\title{
Realization theory of discrete-time linear switched systems
}

\author{
Mihály Petreczky *Laurent Bako ${ }^{\dagger}$ and Jan H. van Schuppen ${ }^{\ddagger}$
}

\begin{abstract}
The paper presents realization theory of discrete-time linear switched systems (abbreviated by DTLSSs). We present necessary and sufficient conditions for an input-output map to admit a discrete-time linear switched state-space realization. In addition, we present a characterization of minimality of discrete-time linear switched systems in terms of reachability and observability. Further, we prove that minimal realizations are unique up to isomorphism. We also discuss algorithms for converting a linear switched system to a minimal one and for constructing a state-space representation from input-output data. The paper uses the theory of rational formal power series in non-commutative variables.
\end{abstract}

Keywords: hybrid systems, switched systems, realization theory, minimal realization.

\section{Introduction}

In this paper we develop realization theory of discrete-time linear switched systems (abbreviated by DTLSSs). DTLSSs are one of the simplest and best studied classes of hybrid systems, [30]. A DTLSS is a discrete-time switched system, such that the continuous sub-system associated with each discrete state is linear. The switching signal is viewed as an external input, and all linear systems live on the same inputoutput- and state-space.

Realization theory. Realization theory is one of the central topics of system theory. For DTLSSs, the subject of realization theory is to answer the following questions.

- When is it possible to construct a (preferably minimal) DTLSS state-space representation of the specified input/output behavior?

- How to characterize minimal DTLSSs which generate the specified input/output behavior?

\footnotetext{
*Univ Lille Nord de France, F-59000 Lille, France, and EMDouai, IA, F-59500 Douai, France, mihaly.petreczky@mines-douai.fr.

${ }^{\dagger}$ Univ Lille Nord de France, F-59000 Lille, France, and EMDouai, IA, F-59500 Douai, France, laurent.bako@mines-douai.fr.

${ }^{\ddagger}$ Centrum Wiskunde en Informatica (CWI) P.O.Box 94079, 1090GB Amsterdam, The Netherlands J.H.van.Schuppen@cwi.nl
} 
Motivation. While there is a substantial literature on linear switched systems, realization theory was addressed only for the continuous-time case [20, 19]. The motivation for devoting a separate paper to realization theory of discrete-time DTLSSs is the following.

1. Realization theory for DTLSSs is substantially different from realization theory for linear systems.

2. Realization theory for DTLSSs is substantially different from the continuoustime case. More precisely, the realization problem both for continuous-time linear switched systems and for DTLSSs can be transformed to the same realization problem for formal power series. The difference lies in the specific transformation.

3. Formulating realization theory explicitly for discrete-time DTLSSs will be useful the identification of these systems. In fact, the results of this paper were already used in [21] for analyzing identifiability of DTLSSs .

Intuitively, the main difference between linear realization theory and that of linear switched systems is the following. For linear switched systems, the realization problem is equivalent to the problem of representing a sequence of numbers (Markovparameters) as products of several non-commuting matrices (pre- and post-multiplied by fixed matrices). For linear case, the corresponding problem involves not products of non-commuting matrices, but powers of one matrix. In addition, for linear switched systems we allow arbitrary non-zero initial state. The presence of a non-zero initial state means that the input response and initial-state response have to be decoupled. A similar approach was already described in [31] for linear systems.

Contribution of the paper We prove that span-reachability and observability of DTLSSs is equivalent to minimality and that minimal realizations are isomorphic. We also show that any DTLSS can be transformed to a minimal one while preserving its input-output behavior, by presenting a minimization algorithm. In addition, we formulate the concept of Markov-parameters and Hankel-matrix for DTLSSs . We show that an input-output map can be realized by a DTLSS if and only if the Hankel-matrix is of finite rank. We also present a procedure for constructing a DTLSS state-space representation from the Hankel-matrix. Our main tool is the theory of rational formal power series [5, 29].

Related work To the best of our knowledge, the results of this paper are new. The results on minimality of DTLSSs were already announced in [21], but no detailed proof was provided. The results on existence of a realization by a DTLSS were not previously published.

The realization problem for hybrid systems was first formulated in [11]. In [17, 35] the relationship between input-output equations and the state-space representations was studied. In [18, 26, 22] realization theory for various classes of hybrid systems were developed. In particular, realization theory for continuous-time (bi)linear switched systems was developed in [20, 19]. The approach of the present paper is similar to that of [20], however the details of the steps are different. There is a vast literature on topics related to realization theory, such as system identification, observability and reachability of hybrid systems, see [16, 6, 30, 2, 1, 33, 34, 32, 14, 27, 4, 8, 15, 35, 17]. 
Our main tool for developing realization theory of DTLSSs is the theory of rational formal power series. This theory was already used for realization theory of nonlinear and multi-dimensional systems, [9, 12, 29, 3]. State-affine systems from [29] include autonomous DTLSSs as a special case. Realization theory of state-affine systems is equivalent to that of rational formal power series. In this paper we reduce the realization problem for DTLSSs directly to that of rational formal power series. Hence, indirectly we also show that the realization problems for DTLSSs and state-affine systems are equivalent. One could probably reduce the realization problem for DTLSSs to that of state-affine systems directly, however it is unclear if such a reduction would be more advantageous.

Outline $\$ 2$ presents a brief overview of realization theory of discrete-time linear systems. $\$ 3$ presents the formal definition of DTLSSs and it formulates the major system-theoretic concepts for this system class. $\$ 4-\$ 5$ states the main results of the paper. $\sqrt{6}$ contains the necessary background on the theory of rational formal power series. The proofs are presented in $\$ 7$ and Appendix A

Notation Denote by $\mathbb{N}$ the set of natural numbers including 0 . The notation described below is standard in automata theory, see [10, 7]. Consider a set $X$ which will be called the alphabet. Denote by $X^{*}$ the set of finite sequences of elements of $X$. Finite sequences of elements of $X$ are be referred to as strings or words over $X$. Each non-empty word $w$ is of the form $w=a_{1} a_{2} \cdots a_{k}$ for some $a_{1}, a_{2}, \ldots, a_{k} \in X$. The element $a_{i}$ is called the ith letter of $w$, for $i=1, \ldots, k$ and $k$ is called the length $w$. We denote by $\varepsilon$ the empty sequence (word). The length of word $w$ is denoted by $|w|$;note that $|\varepsilon|=0$. We denote by $X^{+}$the set of non-empty words, i.e. $X^{+}=X^{*} \backslash\{\varepsilon\}$. We

denote by $w v$ the concatenation of word $w \in X^{*}$ with $v \in X^{*}$. We use the notation of [13] for matrices indexed by sets other than natural numbers. For each $j=1, \ldots, m, e_{j}$ is the $j$ th unit vector of $\mathbb{R}^{m}$, i.e. $e_{j}=\left(\delta_{1, j}, \ldots, \delta_{n, j}\right), \delta_{i, j}$ is the Kronecker symbol.

\section{Realization theory for linear systems}

In this section we present a brief review of realization theory of discrete-time linear systems, based on [31]. Although the results of this section are not used in the paper, they help to get an intuition for the results on realization theory of DTLSSs .

The input-output maps of interest are of the form $y:\left(\mathbb{R}^{m}\right)^{+} \rightarrow \mathbb{R}^{p}$. For each sequence $u=u_{0} \cdots u_{t}, t \geq 0, y(u)$ is the output of the underlying system at time $t$, if inputs $u_{0}, \ldots, u_{t}$ are fed. It is well-known that for $y$ to be realizable by a linear system, it must be of the form

$$
y\left(u_{0} \cdots u_{t}\right)=K_{t}+\sum_{j=0}^{t-1} H_{t-j-1} u_{j}
$$

for some matrices $K_{k} \in \mathbb{R}^{p}, H_{k} \in \mathbb{R}^{p \times m}, k=0,1,2, \ldots$, and for any sequence of inputs $u_{0}, \ldots, u_{t} \in \mathbb{R}^{m}$. Consider a discrete-time linear system

$$
\Sigma\left\{\begin{array}{l}
x_{t+1}=A x_{t}+B u_{t} \text { where } x_{0} \text { is fixed } \\
y_{t}=C x_{t}
\end{array}\right.
$$


where $A, B$ and $C$ are $n \times n, n \times m$ and $p \times n$ real matrices and $x_{0} \in \mathbb{R}^{n}$ is the initial state. Note that the initial state is $x_{0}$, and $x_{0}$ need not be zero. The map $y$ is said to be realized by $\Sigma$, if the output response of $\Sigma$ to any input $u$ equals $y(u)$. This is the case if and only if $y$ is of the form (1), and $K_{t}=C A^{t} x_{0}, H_{t}=C A^{t} B, t \geq 0$. We call $\Sigma$ a minimal realization of $y$, if it has the smallest state-space dimension among all the linear system realizations of $y$.

Theorem 1 ([31]). Assume that $\Sigma$ is a linear system realization of $y$. Then $\Sigma$ is a minimal realization of $y$, if and only if it is weak-reachable and observable. Recall that $\Sigma$ is weak-reachable if and only if $\left(A,\left[\begin{array}{ll}x_{0} & B\end{array}\right]\right)$ is a reachable pair. All minimal realizations of $y$ are isomorphic and any realization of $y$ can be transformed to a minimal one.

The transformation to a minimal system can be carried out by first transforming the linear system to a weak-reachable one, and then to an observable one, [31].

Next, we formulate conditions for existence of a linear system realization of $y$. To this end, we assume that $y$ is of the form (1). This assumption is necessary (but not sufficient) for existence of a realization. We call the matrices $M_{t}=\left[\begin{array}{ll}K_{t} & H_{t}\end{array}\right]$, $t \geq 0$ Markov parameters. This terminology is slightly different from the one used in [31]. Note that $y$ is completely determined by the Markov-parameters $\left\{M_{t}\right\}_{t=0}^{\infty}$. In addition, note that we defined the Markov-parameters without assuming the existence of a linear system realization. In fact, we use the Markov-parameters for characterizing the existence of a linear system realization. More precisely, we define the infinite block Hankel-matrix $H_{y}$ of $y$ as follows $H_{y}=\left(H_{i, j}\right)_{i, j=1}^{\infty}, H_{i, j}=M_{i+j-2}$, i.e. the entries of $H_{y}$ are formed by the entries of the Markov-parameters of $y$.

Theorem 2 ([31]). The map y can be realized by a linear system if and only if the rank of $H_{y}$ is finite. If rank $H_{y}=n<+\infty$, then a minimal linear system realization $\Sigma$ of $y$ can be constructed from the columns of $H_{y}$. In particular, this means that rank $H_{y}$ equals the dimension of any minimal linear system which is a realization of $y$.

Procedure 1. The construction of $\Sigma$ from the columns of $H_{y}$ is as follows. Fix a finite basis in the column space of $H_{y}$. Then $x_{0}$ is formed by the coordinates of the first column of $H_{y}$ in this basis, the rth column of the matrix $B$ represents the coordinates of the $r+1$ th column of $H_{y}$ in this basis. The matrix $C$ is the matrix (in the fixed basis) of the linear map which maps each column to the vector formed by its first p entries. Finally, $A$ is the matrix (in the fixed basis) of the linear map which maps the jth column to the $j+(m+1)$ th column, i.e. it maps the block column $\left(M_{i+j-2}\right)_{i=1}^{\infty}$ to the block column $\left(M_{i+j-1}\right)_{i=1}^{\infty}$.

\section{Linear switched systems}

In this section we present the formal definition of DTLSSs along with a number of relevant system-theoretic concepts for DTLSSs .

Definition 1. Recall from [21] that a discrete-time linear switched system (abbreviated by DTLSS), is a discrete-time control system of the form

$$
\Sigma\left\{\begin{array}{l}
x_{t+1}=A_{q_{t}} x_{t}+B_{q_{t}} u_{t} \text { and } x_{0} \text { is fixed } \\
y_{t}=C_{q_{t}} x_{t} .
\end{array}\right.
$$


Here $Q=\{1, \ldots, D\}$ is the finite set of discrete modes, $D$ is a positive integer. For each $t \in \mathbb{N}, q_{t} \in Q$ is the discrete mode, $u_{t} \in \mathbb{R}$ is the continuous input, $y_{t} \in \mathbb{R}^{p}$ is the output at time $t$. Moreover, $A_{q} \in \mathbb{R}^{n \times n}, B_{q} \in \mathbb{R}^{n \times m}, C_{q} \in \mathbb{R}^{p \times n}$ are the matrices of the linear system in mode $q \in Q$, and $x_{0}$ is the initial continuous state. We will use

$$
\left(p, m, n, Q,\left\{\left(A_{q}, B_{q}, C_{q}\right) \mid q \in Q\right\}, x_{0}\right)
$$

as a short-hand notation for DTLSSs of the form (3).

Throughout the section, $\Sigma$ denotes a DTLSS of the form (3). The inputs of $\Sigma$ are the continuous inputs $\left\{u_{t}\right\}_{t=0}^{\infty}$ and the switching signal $\left\{q_{t}\right\}_{t=0}^{\infty}$. The state of the system at time $t$ is $x_{t}$. Note that any switching signal is admissible. We use the following notation for the inputs of $\Sigma$.

Notation 1 (Hybrid inputs). Denote $\mathscr{U}=Q \times \mathbb{R}^{m}$.

We denote by $\mathscr{U}^{*}$ (resp. $\mathscr{U}^{+}$) the set of all finite (resp. non-empty and finite) sequences of elements of $\mathscr{U}$. A sequence

$$
w=\left(q_{0}, u_{0}\right) \cdots\left(q_{t}, u_{t}\right) \in \mathscr{U}^{+}, t \geq 0
$$

describes the scenario, when the discrete mode $q_{i}$ and the continuous input $u_{i}$ are fed to $\Sigma$ at time $i$, for $i=0, \ldots, t$.

Definition 2 (State and output). Consider a state $x_{\text {init }} \in \mathbb{R}^{n}$. For any $w \in \mathscr{U}^{+}$of the form (4), denote by $x_{\Sigma}\left(x_{\text {init }}, w\right)$ the state of $\Sigma$ at time $t+1$, and denote by $y_{\Sigma}\left(x_{\text {init }}, w\right)$ the output of $\Sigma$ at time $t$, if $\Sigma$ is started from $x_{\text {init }}$ and the inputs $\left\{u_{i}\right\}_{i=0}^{t}$ and the discrete modes $\left\{q_{i}\right\}_{i=0}^{t}$ are fed to the system. For notational purposes, we define $x_{\Sigma}\left(x_{i n i t}, \varepsilon\right)=$ $x_{\text {init }}$.

That is, $x_{\Sigma}\left(x_{\text {init }}, w\right)$ is defined recursively as follows; $x_{\Sigma}\left(x_{\text {init }}, \varepsilon\right)=x_{\text {init }}$, and if $w=$ $v(q, u)$ for some $(q, u) \in \mathscr{U}, v \in \mathscr{U}^{*}$, then

$$
x_{\Sigma}\left(x_{i n i t}, w\right)=A_{q} x_{\Sigma}\left(x_{i n i t}, v\right)+B_{q} u .
$$

If $w \in \mathscr{U}^{+}$and $w=v(q, u),(q, u) \in \mathscr{U}, v \in \mathscr{U}^{*}$, then

$$
y_{\Sigma}\left(x_{\text {init }}, w\right)=C_{q} x_{\Sigma}\left(x_{\text {init }}, v\right) .
$$

Definition 3 (Input-output map). The map $y_{\Sigma}: \mathscr{U}^{+} \rightarrow \mathbb{R}^{p}$, defined by $\forall w \in \mathscr{U}^{+}$: $y_{\Sigma}(w)=y\left(x_{0}, w\right)$, is called the input-output map of $\Sigma$.

That is, the input-output map of $\Sigma$ maps each sequence $w \in \mathscr{U}^{+}$to the output generated by $\Sigma$ under the hybrid input $w$, if started from the initial state $x_{0}$. The definition above implies that the input-output behavior of a DTLSS can be formalized as a map

$$
f: \mathscr{U}^{+} \rightarrow \mathbb{R}^{p} .
$$

The value $f(w)$ for $w$ of the form (4) represents the output of the underlying black-box system at time $t$, if the continuous inputs $\left\{u_{i}\right\}_{i=0}^{t}$ and the switching sequence $\left\{q_{i}\right\}_{i=0}^{t}$ are fed to the system. This black-box system may or may not admit a description by a DTLSS.

Next, we define when a general map $f$ of the form (5) is adequately described by the DTLSS $\Sigma$, i.e. when $\Sigma$ is a realization of $f$. 
Definition 4 (Realization). The DTLSS $\Sigma$ is a realization of an input-output map $f$ of the form (5), if $f$ equals the input-output map of $\Sigma$, i.e. $f=y_{\Sigma}$.

The reachable set $\operatorname{Reach}(\Sigma)$ of $\Sigma$ is the set of all states which can be reached from the initial state $x_{0}$ of $\Sigma$, i.e.

$$
\operatorname{Reach}(\Sigma)=\left\{x_{\Sigma}\left(x_{0}, w\right) \in \mathbb{R}^{n} \mid w \in \mathscr{U}^{*}\right\}
$$

Definition 5 ((Span-)Reachability)). The DTLSS $\Sigma$ is reachable, if Reach $(\Sigma)=\mathbb{R}^{n}$, and $\Sigma$ is span-reachable if $\mathbb{R}^{n}$ is the smallest vector space containing Reach $(\Sigma)$.

Reachability implies span-reachability but in general they are not equivalent.

Definition 6 (Observability). The DTLSS $\Sigma$ is called observable if for any two states $x_{1}, x_{2} \in \mathbb{R}^{n}$ of $\Sigma$,

$$
\left(\forall w \in \mathscr{U}^{+}: y_{\Sigma}\left(x_{1}, w\right)=y_{\Sigma}\left(x_{2}, w\right)\right) \Longrightarrow x_{1}=x_{2}
$$

That is, observability means that if we pick any two states of the system, then for some continuous input and switching signal, the resulting outputs will be different.

Definition 7 (Dimension). The dimension of $\Sigma$, denoted by $\operatorname{dim} \Sigma$, is the dimension $n$ of its state-space.

Note that the number of discrete states is fixed, and hence it is not included into the definition of dimension. The reason for this is the following. We are interested in realizations of input-output maps, which map continuous inputs and switching signals to continuous outputs. Hence, for all possible DTLSS realizations, the set of discrete modes is fixed.

Definition 8 (Minimality). Let $f$ be an input-output map. Then $\Sigma$ is a minimal realization of $f$, if $\Sigma$ is a realization of $f$, and for any DTLSS $\hat{\Sigma}$ which is a realization of $f$, $\operatorname{dim} \Sigma \leq \operatorname{dim} \hat{\Sigma}$.

Definition 9 (DTLSS morphism). Consider a DTLSS $\Sigma_{1}$ of the form (3) and a DTLSS $\Sigma_{2}$ of the form

$$
\Sigma_{2}=\left(p, m, n^{a}, Q,\left\{\left(A_{q}^{a}, B_{q}^{a}, C_{q}^{a}\right) \mid q \in Q\right\}, x_{0}^{a}\right)
$$

Note that $\Sigma_{1}$ and $\Sigma_{2}$ have the same set of discrete modes. A matrix $\mathscr{S} \in \mathbb{R}^{n^{a} \times n}$ is said to be a DTLSS morphism from $\Sigma_{1}$ to $\Sigma_{2}$, denoted by $\mathscr{S}: \Sigma_{1} \rightarrow \Sigma_{2}$, if

$$
\mathscr{S} x_{0}=x_{0}^{a} \text {, and } \forall q \in Q: A_{q}^{a} \mathscr{S}=\mathscr{S} A_{q}, B_{q}^{a}=\mathscr{S} B_{q}, C_{q}^{a} \mathscr{S}=C_{q} .
$$

The morphism $\mathscr{S}$ is called surjective (injective) if $\mathscr{S}$ is surjective (injective) as a linear map. The morphism $\mathscr{S}$ is said to be a DTLSS isomorphism, if it is an isomorphism as a linear map. 


\section{Main result on minimality}

Below we present the main results of the paper on minimality of DTLSSs. In addition, we present a minimization procedure and rank tests for checking minimality. In the sequel, $\Sigma$ denotes a DTLSS of the form (3), and $f$ denotes an input-output map $f$ : $\mathscr{U}^{+} \rightarrow \mathbb{R}^{p}$.

Theorem 3 (Minimality). 1. A DTLSS realization of $f$ is minimal, if and only if it is span-reachable and observable.

2. All minimal DTLSS realizations of $f$ are isomorphic.

3. Every DTLSS realization of $f$ can be converted to a minimal DTLSS realization of $f$ (see Procedure 4 below).

The proof of Theorem 3 is presented in 87

Remark 1. Note that $\Sigma$ can be minimal, while none of the linear subsystems is minimal, see Example 1 below. Since all minimal realizations are isomorphic, it then follows that such a DTLSS cannot be transformed to a one where at least one subsystem is minimal without loosing input-output behavior.

For analogous theorem for continuous-time linear switched systems see [20, 19]. Intuitively, the theorem says the following. First, a minimal DTLSS should not contain states which are not linear combination of the reachable ones (hence span-reachability). Second, a minimal DTLSS should not contain multiple states which exhibit the same input-output behavior (hence observability). Next, we present rank conditions for observability and span-reachability. These conditions can be used to test minimality and to formulate Procedure 4

Notation 2. Let $X$ be a finite set, $\mathscr{X}$ be a linear space, $A_{\sigma}: \mathscr{X} \rightarrow \mathscr{X}, \sigma \in X$ be linear maps and let $w \in X^{*}$. The linear map $A_{w}$ on $\mathscr{X}$ is defined as follows. If $w=\varepsilon$, then $A_{\varepsilon}$ is the identity map, i.e $A_{\varepsilon} x=x$ for all $x \in \mathscr{X}$. If $w=\sigma_{1} \sigma_{2} \cdots \sigma_{k} \in X^{*}, \sigma_{1}, \cdots \sigma_{k} \in X$, $k>0$, then

$$
A_{w}=A_{\sigma_{k}} A_{\sigma_{k-1}} \cdots A_{\sigma_{1}} .
$$

If $\mathscr{X}=\mathbb{R}^{n}$ for some $n>0$, then $A_{w}$ and each $A_{\sigma}, \sigma \in X$ can be identified with an $n \times n$ matrix. In this case $A_{w}$ defines a product of matrices.

We denote by $Q^{<n}$ the set $\left\{w \in Q^{*}|| w \mid<n\right\}$ of all words $w \in Q^{*}$ of length at most $n-1$. We denote by $M_{n}$ the cardinality of $Q^{<n}$ and we fix an enumeration

$$
Q^{<n}=\left\{v_{1}, \ldots, v_{M_{n}}\right\} .
$$

We will use the notation defined above to define observability and reachability matrices for DTLSSs .

Theorem 4. Span-Reachability. Define the span-reachability matrix $R(\Sigma)$ of $\Sigma$

$$
\begin{aligned}
& \mathscr{R}(\Sigma)=\left[\begin{array}{llll}
A_{v_{1}} \widetilde{B}, & A_{v_{2}} \widetilde{B}, & \cdots, & A_{v_{M_{n}}} \widetilde{B}
\end{array}\right] \in \mathbb{R}^{n \times(|Q| m+1) M_{n}} \text { where } \\
& \widetilde{B}=\left[x_{0}, \quad B_{1}, \cdots, B_{D}\right]
\end{aligned}
$$


Then $\Sigma$ is span-reachable if and only if $\operatorname{rank} \mathscr{R}(\Sigma)=n$.

Observability. Define the observability matrix $O(\Sigma) \in \mathbb{R}^{p|Q| M_{n} \times n}$ of $\Sigma$ as follows.

$$
O(\Sigma)=\left[\begin{array}{c}
\widetilde{C} A_{v_{1}} \\
\widetilde{C} A_{v_{2}} \\
\vdots \\
\widetilde{C} A_{v_{M_{n}}}
\end{array}\right] \text { where } \widetilde{C}=\left[\begin{array}{c}
C_{1} \\
C_{2} \\
\vdots \\
C_{D}
\end{array}\right]
$$

Then $\Sigma$ is observable if and only if rank $O(\Sigma)=n$.

Informally, $\mathscr{R}(\Sigma)$ is formed by horizontal concatenation of blocks $A_{w} B_{q}$, for all $w \in Q^{<n}, q \in Q$, and $O(\Sigma)$ is the vertical concatenation of blocks $C_{q} A_{w}, q \in Q, w \in Q^{<n}$. Notice that if $Q=\{1\}$, then $\mathscr{R}(\Sigma)$ is the controllability matrix of $\left(A_{1},\left[\begin{array}{ll}x_{0} & B_{1}\end{array}\right]\right)$ and $O(\Sigma)$ is the observability matrix of $\left(C_{1}, A_{1}\right)$. Hence, the linear system $\left(A_{1}, B_{1}, C_{1}, x_{0}\right)$ is weak-reachable (observable) if and only if it is span-reachable (observable), if interpreted as a DTLSS. Hence, Theorem 3 implies Theorem 1

The result of Theorem 4 follow from [30], the detailed proof can be found in Appendix A Next, we formulate procedures for reachability, observability and minimality reduction of DTLSSs .

Procedure 2 (Reachability reduction). Assume $\operatorname{dim} \mathscr{R}(\Sigma)=n^{r}$ and choose a basis $b_{1}, \ldots, b_{n}$ of $\mathbb{R}^{n}$ such that $b_{1}, \ldots, b_{n^{r}}$ span $\operatorname{Im} \mathscr{R}(\Sigma)$. In the new basis, $A_{q}, B_{q}, C_{q}, q \in Q$ and $x_{0}$ become as follows

$$
A_{q}=\left[\begin{array}{cc}
A_{q}^{r}, & A_{q}^{\prime} \\
0, & A_{q}^{\prime \prime}
\end{array}\right], C_{q}=\left[\begin{array}{ll}
C_{q}^{r}, & C_{q}^{n r}
\end{array}\right], B_{q}=\left[\begin{array}{c}
B_{q}^{r} \\
0
\end{array}\right], x_{0}=\left[\begin{array}{c}
x_{0}^{r} \\
0
\end{array}\right]
$$

where $A_{q}^{r} \in \mathbb{R}^{n^{r} \times n^{r}}, B_{q}^{r} \in \mathbb{R}^{n^{r} \times m}, x_{0}^{r} \in \mathbb{R}^{n_{r}}$. Then $\Sigma_{r}=\left(p, m, n^{r}, Q,\left\{\left(A_{q}^{r}, B_{q}^{r}, C_{q}^{r}\right) \mid q \in\right.\right.$ $\left.Q\}, x_{0}^{r}\right)$ is span-reachable, and has the same input-output map as $\Sigma$.

Intuitively, $\Sigma_{r}$ is obtained from $\Sigma$ by restricting the dynamics and the output map of $\Sigma$ to the space $\operatorname{Im} R(\Sigma)$.

Procedure 3 (Observability reduction). Assume that $\operatorname{ker} O(\Sigma)=n-n^{o}$ and let $b_{1}, \ldots, b_{n}$ be a basis in $\mathbb{R}^{n}$ such that $b_{n^{o}+1}, \ldots, b_{n}$ span $\operatorname{ker} O(\Sigma)$. In this new basis, $A_{q}, B_{q}, C_{q}$ and $x_{0}$ can be rewritten as

$$
A_{q}=\left[\begin{array}{cc}
A_{q}^{o}, & 0 \\
A_{q}^{\prime}, & A_{q}^{\prime \prime}
\end{array}\right], C_{q}=\left[\begin{array}{cc}
C_{q}^{o}, & 0
\end{array}\right], B_{q}=\left[\begin{array}{c}
B_{q}^{o} \\
B_{q}^{\prime}
\end{array}\right], x_{0}=\left[\begin{array}{l}
x_{0}^{o} \\
x_{0}^{\prime}
\end{array}\right]
$$

where $A_{q}^{o} \in \mathbb{R}^{n^{o} \times n^{o}}, B_{q}^{o} \in \mathbb{R}^{n^{o} \times m}, C_{q}^{o} \in \mathbb{R}^{p \times n^{o}}$ and $x_{0}^{o} \in \mathbb{R}^{n_{o}}$. Then the DTLSS $\Sigma_{o}=$ $\left(p, m, n^{o}, Q,\left\{\left(A_{q}^{o}, B_{q}^{o}, C_{q}^{o}\right) \mid q \in Q\right\}, x_{0}^{o}\right)$ is observable and its input-output map is the same as that of $\Sigma$. If $\Sigma$ is span-reachable, then so is $\Sigma_{o}$.

Intuitively, $\Sigma_{o}$ is obtained from $\Sigma$ by merging any two states $x_{1}, x_{2}$ of $\Sigma$, for which $O(\Sigma) x_{1}=O(\Sigma) x_{2}$. The latter is equivalent to $y_{\Sigma}\left(x_{1}, w\right)=y_{\Sigma}\left(x_{2}, w\right), \forall w \in \mathscr{U}^{+}$. 
Procedure 4 (Minimization). First transform $\Sigma$ to a span-reachable DTLSS $\Sigma_{r}$ and then transform $\Sigma_{r}$ to an observable DTLSS $\Sigma_{m}=\left(\Sigma_{r}\right)_{o}$. Then $\Sigma_{m}$ is a minimal realization of the input-output map of $\Sigma$.

The correctness of Procedures 23 and 4 are proved in $\$ 7$ using the theory of formal power series. Note that the correctness of Procedure 3 and of Procedure 2 (in case of $x_{0}=0$ ) has already been shown by a direct proof in [30].

Example 1. Let $\Sigma=\left(p, m, n, Q,\left\{\left(A_{q}, B_{q}, C_{q}\right) \mid q \in Q\right\}, x_{0}\right)$ with $Q=\{1,2\}, n=3$, $x_{0}=\left[\begin{array}{lll}0 & 1 & 0\end{array}\right]^{T}$,

$$
\begin{aligned}
& A_{1}=\left[\begin{array}{lll}
0 & 1 & 0 \\
0 & 0 & 1 \\
0 & 0 & 1
\end{array}\right], B_{1}=\left[\begin{array}{l}
0 \\
0 \\
0
\end{array}\right], C_{1}=\left[\begin{array}{lll}
1 & 0 & 0
\end{array}\right] \\
& A_{2}=\left[\begin{array}{lll}
0 & 1 & 0 \\
0 & 1 & 1 \\
0 & 0 & 1
\end{array}\right], B_{2}=\left[\begin{array}{l}
0 \\
1 \\
0
\end{array}\right], C_{2}=\left[\begin{array}{lll}
0 & 0 & 1
\end{array}\right]
\end{aligned}
$$

This system is observable, but it is not span-reachable. In order to see observability, notice that the sub-matrix $\left[\begin{array}{lll}C_{1}^{T} & \left(C_{1} A_{1}\right)^{T} & C_{2}^{T}\end{array}\right]^{T}$ of $O(\Sigma)$ is of rank 3 . In order to see that $\Sigma$ is not span-reachable, notice that if $(x, y, z)^{T}$ is a column of $R(\Sigma)$, then $z=0$. Hence $\operatorname{dim} R(\Sigma) \leq 2$.

Using Procedure 4 we can transform $\Sigma$ to the minimal realization

$$
\Sigma_{m}=\left(p, m, n^{m}, Q,\left\{\left(A_{q}^{m}, B_{q}^{m}, C_{q}^{m}\right) \mid q \in Q\right\}, x_{0}^{m}\right)
$$

of $y_{\Sigma}: Q=\{1,2\}, n^{m}=2, x_{0}^{m}=[1,0]^{T}$ and

$$
\begin{aligned}
& A_{1}^{m}=\left[\begin{array}{ll}
0 & 0 \\
1 & 0
\end{array}\right], B_{1}^{m}=\left[\begin{array}{l}
0 \\
0
\end{array}\right], C_{1}^{m}=\left[\begin{array}{ll}
0, & 1
\end{array}\right] \\
& A_{2}^{m}=\left[\begin{array}{ll}
1 & 0 \\
1 & 0
\end{array}\right], B_{2}^{m}=\left[\begin{array}{l}
1 \\
0
\end{array}\right], C_{2}^{m}=\left[\begin{array}{ll}
0, & 0
\end{array}\right]
\end{aligned}
$$

Using [31], it is easy to see that neither $\left(A_{1}^{m}, B_{1}^{m}, C_{1}^{m}, x_{0}^{m}\right)$ nor $\left(A_{2}^{m}, B_{2}^{m}, C_{2}^{m}, x_{0}^{m}\right)$ are minimal.

\section{Main results on existence of a realization}

We present the necessary and sufficient conditions for the existence of a DTLSS realization for an input-output map. In the sequel, $f$ denotes a map of the form (5). To this end, we need the notion of the Hankel-matrix and Markov-parameters of an inputoutput map. More precisely, we proceed as follows. First, we define the notion of Markov parameters of $f$ and use them to define the Hankel-matrix of $f$. We then use the Hankel-matrix to formulate conditions for existence of a DTLSS realization of $f$. To this end, we need the following notation. 
Notation 3. In the sequel, we identify any element $w=\left(q_{0}, u_{0}\right) \cdots\left(q_{t}, u_{t}\right) \in \mathscr{U}^{+}$with the pair of sequences $(v, u), v \in Q^{+}, u \in\left(\mathbb{R}^{m}\right)^{+}, v=q_{0} \cdots q_{t}$ and $u=u_{0} \cdots u_{t}$.

Notation 4. Consider the input-output map $f$. For each word $v \in Q^{+}$of length $|v|=$ $t>0$ define $f_{v}:\left(\mathbb{R}^{m}\right)^{t} \rightarrow \mathbb{R}^{p}$ as

$$
f_{v}(u)=f((v, u)) .
$$

Now we are ready to define the Markov-parameters of an input-output map.

Definition 10 (Markov-parameters). Denote $Q^{k, *}=\left\{w \in Q^{*}|| w \mid \geq k\right\}$. Define the maps $S_{0}^{f}: Q^{1, *} \rightarrow \mathbb{R}^{p}$ and $S_{j}^{f}: Q^{2, *} \rightarrow \mathbb{R}^{p}, j=1, \ldots, m$ as follows; for any $v \in Q^{*}$, $q, q_{0} \in Q$,

$$
\begin{aligned}
& S_{0}^{f}(v q)=f_{v q}(0, \ldots, 0) \text { and } \\
& S_{j}^{f}\left(q_{0} v q\right)=f_{q_{0} v q}\left(e_{j}, 0, \ldots, 0\right)-f_{q_{0} v q}(0, \ldots, 0),
\end{aligned}
$$

with $e_{j} \in \mathbb{R}^{m}$ is the vector with 1 as its $j$ th entry and zero everywhere else. The collection of maps $\left\{S_{j}^{f}\right\}_{j=0}^{m}$ is called the Markov-parameters of $f$.

The function $S_{0}^{f}$ can be viewed as the initial state-response and the functions $S_{j}^{f}$, $j=1, \ldots, m$ can be viewed as input responses. The interpretation of $S_{0}^{f}, S_{j}^{f}$ will become more clear after we define the concept of a generalized convolution representation. Note that the values of the Markov-parameters can be obtained from the values of $f$, i.e. by means of input-output experiments.

Notation 5 (Sub-word). Consider the sequence $v=q_{0} \cdots q_{t} \in Q^{+}, q_{0}, \ldots, q_{t} \in Q, t \geq 0$. For each $j, k \in\{0, \ldots, t\}$, define the word $v_{j \mid k} \in Q^{*}$ as follows; if $j>k$, then $v_{j \mid k}=\varepsilon$, if $j=k$, then $v_{j \mid j}=q_{j}$ and if $j<k$, then $v_{j \mid k}=q_{j} q_{j+1} \cdots q_{k}$. That is, $v_{j \mid k}$ is the sub-word of $v$ formed by the letters from the jth to the kth letter.

Definition 11 (Convolution representation). The input-output map $f$ has a generalized convolution representation (abbreviated as GCR), if for all $w=(v, u) \in \mathscr{U}^{+}$, $v=q_{0} \cdots q_{t}, u=u_{0} \cdots u_{t}, q_{0}, \ldots, q_{t} \in Q, u_{0}, \ldots u_{t} \in \mathbb{R}^{m}, f(w)$ can be expressed via the Markov-parameters of $f$ as follows.

$$
f(w)=S_{0}^{f}\left(v_{0 \mid t-1} q_{t}\right)+\sum_{k=0}^{t-1} S^{f}\left(q_{k} v_{k+1 \mid t-1} q_{t}\right) u_{k}
$$

where $S^{f}(w)=\left[S_{1}^{f}(w), \quad S_{2}^{f}(w), \quad \ldots, \quad S_{m}^{f}(w)\right] \in \mathbb{R}^{p \times m}$ for all $w \in Q^{*}$.

Remark 2. If $f$ has a GCR, then the Markov-parameters of $f$ determine $f$ uniquely.

The motivation for introducing GCRs is that existence of a GCR is a necessary condition for realizability by DTLSSs. More precisely, the following holds.

Lemma 1. The map $f$ is realized by the DTLSS $\Sigma$ if and only if $f$ has a GCR and for all $v \in Q^{*}, q, q_{0} \in Q$,

$$
\begin{aligned}
& S_{0}^{f}(v q)=C_{q} A_{v} x_{0} \text { and } \\
& S_{j}^{f}\left(q_{0} v q\right)=C_{q} A_{v} B_{q_{0}} e_{j}, j=1, \ldots, m .
\end{aligned}
$$


The proof of Lemma 1 can be found in Appendix A From Lemma 1 it follows that if $f$ is realizable by a DTLSS, then the values of $S_{0}^{f}$ and $S_{j}^{f}, j=1, \ldots, m$ can be expressed as products of matrices. Moreover, $S_{0}^{f}$ corresponds to the part of the response which depends on the initial state, and $\left\{S_{j}^{f}\right\}_{j=1}^{m}$ encodes the response from the zero initial state.

We can draw the following analogy with the linear case $\$ 2$ Existence of a GCR is analogous to the requirement that the input-output map is of the form (11). The Markovparameter $S_{0}^{f}(v q)$ corresponds to the vector $K_{|v|}$, and the vector $S_{j}^{f}\left(q_{0} v q\right)$ corresponds to the $j$ th column of the matrix $H_{|v|}$. Finally, if $f$ can be realized by a DTLSS, then the Markov-parameters can be expressed as products of matrices (9). This is analogous to the linear case, where $K_{t}=C A^{t} x_{0}$ and $H_{t}=C A^{t} B$ holds for $t \geq 0$, if $\left(A, B, C, x_{0}\right)$ is a realization of the input-output map. In fact,if $Q=\{1\}$, i.e. we are dealing with linear systems, then $S_{0}^{f}(v q)=K_{|v|}, S_{j}^{f}\left(q_{0} v q\right)$ is the $j$ th column of $H_{|v|}$ and the GCR is the representation of the form (1), and the right-hand sides of (9) becomes $C A^{|v|} x_{0}$, $C A^{|v|} B e_{j}$, where $C=C_{1}, A=A_{1}, B=B_{1}$.

Next, we define the concept of a Hankel-matrix. Similarly to the linear case, the entries of the Hankel-matrix are formed by the Markov parameters. For the definition of the Hankel-matrix of $f$, we will use lexicographical ordering on the set of sequences $Q^{*}$.

Remark 3 (Lexicographic ordering). Recall that $Q=\{1, \ldots, D\}$. We define a lexicographic ordering $\prec$ on $Q^{*}$ as follows. For any $v, s \in Q^{*}, v \prec s$ if either $|v|<|s|$ or $0<|v|=|s|, v \neq s$ and for some $l \in\{1, \ldots,|s|\}, v_{l}<s_{l}$ with the usual ordering of integers and $v_{i}=s_{i}$ for $i=1, \ldots, l-1$. Here $v_{i}$ and $s_{i}$ denote the ith letter of $v$ and $s$ respectively. Note that $\prec$ is a complete ordering and $Q^{*}=\left\{v_{1}, v_{2}, \ldots\right\}$ with $v_{1} \prec v_{2} \prec \ldots$ Note that $v_{1}=\varepsilon$ and for all $i \in \mathbb{N}, q \in Q, v_{i} \prec v_{i} q$.

In order to simplify the definition of a Hankel-matrix, we introduce the notion of a combined Markov-parameter.

Definition 12 (Combined Markov-parameters). A combined Markov-parameter $M^{f}(v)$ of $f$ indexed by the word $v \in Q^{*}$ is the following $p D \times(D m+1)$ matrix

$$
M^{f}(v)=\left[\begin{array}{cccc}
S_{0}^{f}(v 1), & S^{f}(1 v 1), & \cdots, & S^{f}(D v 1) \\
S_{0}^{f}(v 2), & S^{f}(1 v 2), & \cdots, & S^{f}(D v 2) \\
\vdots & \vdots & \cdots & \vdots \\
S_{0}^{f}(v D), & S^{f}(1 v D), & \cdots, & S^{f}(D v D)
\end{array}\right]
$$

where for any $w \in Q^{+},|w|>2, S^{f}(w)=\left[\begin{array}{llll}S_{1}^{f}(w), & S_{2}^{f}(w), & \ldots, & S_{m}^{f}(w)\end{array}\right]$.

Definition 13 (Hankel-matrix). Consider the lexicographic ordering $\prec$ of $Q^{*}$ from Remark 3 Define the Hankel-matrix $H_{f}$ of $f$ as the following infinite matrix

$$
H_{f}=\left[\begin{array}{ccccc}
M^{f}\left(v_{1} v_{1}\right), & M^{f}\left(v_{2} v_{1}\right), & \ldots, & M^{f}\left(v_{k} v_{1}\right), & \ldots \\
M^{f}\left(v_{1} v_{2}\right), & M^{f}\left(v_{2} v_{2}\right), & \cdots, & M^{f}\left(v_{k} v_{2}\right), & \cdots, \\
M^{f}\left(v_{1} v_{3}\right) & M^{f}\left(v_{2} v_{3}\right), & \cdots, & M^{f}\left(v_{k} v_{3}\right) & \cdots \\
\vdots & \vdots & \ldots & \vdots & \ldots
\end{array}\right],
$$


i.e. the $p D \times(m D+1)$ block of $H_{f}$ in the block row $i$ and block column $j$ equals the combined Markov-parameter $M^{f}\left(v_{j} v_{i}\right)$ of $f$. The rank of $H_{f}$, denoted by rank $H_{f}$, is the dimension of the linear span of its columns.

The Hankel-matrix of $f$ can also be viewed as a matrix rows and columns of which are indexed by words from $Q^{*}$.

Remark 4 (Alternative definition of the Hankel-matrix). Notice that every row index $0<l \in \mathbb{N}$ of $H_{f}$ can be identified with a tuple $(v, i), i=1, \ldots, p D$ and $v \in Q^{*}$ as follows; $v=v_{r}$, i.e. $v$ is the rth element of $Q^{*}$, for some $0<r \in \mathbb{N}$ such that $l=(r-1) D p+i$. In fact the identification above is a one-to-one mapping.

Similarly, every column index $0<k \in \mathbb{N}$ can be identified with a pair $(w, j)$ where $w \in Q^{*}, j \in J_{f}=\{0\} \cup Q \times\{1, \ldots, m\}$, where $w=v_{r}$, i.e. $w$ is the rth element of $Q^{*}$ for some $r \in \mathbb{N}$ such that $k=(r-1)(m D+1)+i$ for some integer $i=1, \ldots, m D+1$, and if $i=1$ then $j=0$ and if $i=m(q-1)+z+1$ for some $q \in Q$ and $z=1, \ldots, m$, then $j=(q, z)$. This identification is one-to-one.

Using the identification of row and column indices outlined above, we can view $H_{f}$ as a matrix, rows of which are indexed by $(v, i), v \in Q^{*}, i=1, \ldots, p D$, and columns of which are indexed by $(w, j), w \in Q^{*}, j \in J_{f}$. The entry $\left[H_{f}\right]_{(v, i),(w, j)}$ of $H_{f}$ indexed by row index $(v, i)$ and column index $(w, j)$ is the ith entry of the rth column of $M^{f}(w v)$, where $r=1$, if $j=0$ and $r=m(q-1)+z+1$ if $j=(q, z)$. In other words,

$$
\begin{aligned}
& {\left[H_{f}\right]_{(v, i),(w,(q, z))}=\left[S_{z}^{f}\left(q w v \alpha_{i}\right)\right]_{l},} \\
& {\left[H_{f}\right]_{(v, i),(w, 0)}=\left[S_{0}^{f}\left(w v \alpha_{i}\right)\right]_{l}}
\end{aligned}
$$

where $\alpha_{i}=K+1$ with $K$ and $l$ defined from $i$ by the decomposition $i=p K+l, K=$ $0,1, \ldots, D-1, l=1, \ldots, p$. Here, $[a]_{l}$ denotes the lth entry of a vector $a$.

It is not difficult to see that for $Q=\{1\}, H_{f}$ is the same as the Hankel-matrix defined in 92 The main result on realization theory of DTLSSs can be stated as follows.

Theorem 5. The map $f$ has a realization by a DTLSS if and only if $f$ has a GCR and rank $H_{f}<+\infty$. A minimal realization of $f$ can be constructed from $H_{f}$ (see Procedure 5) and any minimal DTLSS realization of $f$ has dimension rank $H_{f}$.

Procedure 5. If rank $H_{f}=n<+\infty$, then a DTLSS $\Sigma_{f}$ of the form (3) can be constructed from $H_{f}$ as follows. Choose a basis in the column space of $H_{f}$.

In this basis, let $x_{0}$ be the coordinates of the first column of $H_{f}$. For each $l=$ $1, \ldots, m$, the lth column of $B_{q}, q \in Q$ is formed by coordinates of the $m(q-1)+l+1$ th column of $H_{f}$. Let $C_{q}, q \in Q$ be the matrix of the linear map which maps every column to the vector formed by its rows indexed by $p(q-1)+1, p(q-1)+2, \ldots, p q$. Define $A_{q}, q \in Q$ as the matrix of the linear map which maps the rth column of the block column $\left(M\left(v_{j} v_{i}\right)\right)_{i=1}^{\infty}$ to the rth column of the block column $\left(M\left(v_{j} q v_{i}\right)\right)_{i=1}^{\infty}$, for each $j=1,2, \ldots$, and $r=1,2, \ldots,(D m+1)$.

Alternatively, using Remark 4 we can describe $\Sigma_{f}$ as follows. The initial state $x_{0}$ is formed by the coordinates of the column of $H_{f}$ indexed by $(\varepsilon, 0)$. The lth column of $B_{q}, q \in Q$ is formed by the coordinates of the column of $H_{f}$ indexed by $(\varepsilon,(q, l))$, 
$l=1, \ldots, m$. The matrix $C_{q}, q \in Q$ is the matrix of the linear map which maps each column of $H_{f}$ to the vector formed by its rows which are indexed by $(\varepsilon, p(q-1)+$ $1), \ldots,(\varepsilon, p q)$. Finally, $A_{q}$ is the matrix of the map which maps each column indexed by $(w, j)$ to the column indexed by $(w q, j), w \in Q^{*}, j \in J_{f}$.

Notice that for $Q=\{1\}$, Theorem 5 implies Theorem 2 , and Procedure 5 reduces to Procedure 1.

Example 2. Consider a SISO input-output map $f$ such that for any $v \in Q^{+},|v|=t$,

$$
f_{v}\left(u_{1}, \ldots, u_{t}\right)=\left\{\begin{array}{cc}
1+\sum_{j=1}^{t-2} u_{j} & \text { if } t>1 \text { and } v=2^{t-1} 1 \text { or } \\
v=2^{t-2} 11, \\
0 \quad \text { otherwise }
\end{array}\right.
$$

Hence, the Markov-parameters of $f$ are as follows

$$
\begin{aligned}
& S_{0}^{f}(v)= \begin{cases}1 & \text { if } t>1 \text { and } v=2^{t-1} 1 \text { or } v=2^{t-2} 11 \\
0 & \text { otherwise }\end{cases} \\
& S_{1}^{f}(v)= \begin{cases}1 & \text { if } t>2 \text { and } v=2^{t-1} 1 \text { or } v=2^{t-2} 11 \\
0 & \text { otherwise }\end{cases}
\end{aligned}
$$

It is easy to check that $\Sigma$ from Example $\square$ satisfies (9) from Lemma 17 hence $\Sigma$ is a realization of $f$.

Consider the Hankel-matrix $H_{f}$ of $f$. It is easy to see that the set of columns of $H_{f}$ contains two elements: $b_{1}$ and $b_{2}$. The entries of $b_{1}$ equal 1 , if indexed by $(v, 1)$ with $|v|>0$ and $v=2^{|v|}$ or $v=2^{|v|-1} 1$ and are zero otherwise. The only non-zero entry of $b_{2}$ is 1 and it is indexed by $(\varepsilon, 1)$. Applying Procedure 5 to our example, and taking $\left(b_{1}, b_{2}\right)$ as a basis of $\operatorname{Im} H_{f}$, we obtain a DTLSS of the form (3) which coincides with $\Sigma_{m}$ from Example 1

Indeed, since the column of $H_{f}$ indexed by $(\varepsilon,(1,1))$ is zero, and the column indexed by $(\varepsilon, 0)$ and $(\varepsilon,(2,1))$ is $b_{1}$, we get $B_{1}=0, B_{2}=x_{0}=(1,0)$. Since the entries of any column indexed by $(\varepsilon, 2)$ are zero, we get $C_{2}=0$. Since the entries of $b_{1}$ and $b_{2}$ indexed by $(\varepsilon, 1)$ are 1 , we get $C_{1}=(1,1)^{T}$. Note that if the column of $H_{f}$ indexed by $(w, j)$ equals $b_{1}$, then the column indexed by $(w 1, j)$ equals $b_{2}$, the column indexed by $(w 2, j)$ equals $b_{1}+b_{2}$. If the column indexed by $(w, j)$ equals $b_{2}$, then the column indexed by $(w 1, j)$ and $(w 2, j)$ are both zero. Hence, if $A_{1}$ and $A_{2}$ are viewed as linear maps on $\operatorname{Im} H_{f}$, then $A_{1} b_{1}=b_{2}, A_{1} b_{2}=0, A_{2} b_{2}=0, A_{2} b_{1}=b_{1}+b_{2}$. In other words, the matrices $A_{1}$ and $A_{2}$ are precisely the same as the matrices $A_{1}^{m}$ and $A_{2}^{m}$ from Example [1]

Note that once the Markov-parameters are defined, the definition of Hankel-matrix presented above coincides with that of the continuous-time case. As a consequence, we can repeat the realization algorithm described in [25, Algorithm 1] for DTLSSs . Moreover, [25, Theorem 4] holds for DTLSSs . For the sake of completeness, below we state the realization algorithm and its correctness explicitly for DTLSSs .

Definition $14\left(H_{f, L, M}\right.$ sub-matrices of $\left.H_{f}\right)$. For $L, M \in \mathbb{N}$ define the integers $I_{L}=$ $\mathbf{N}(L) p D$ and $J_{M}=\mathbf{N}(M)(m D+1)$. Denote by $H_{f, L, M}$ the following upper-left $I_{L} \times J_{M}$ 
sub-matrix of $H_{f}$,

$$
\left[\begin{array}{cccc}
M^{f}\left(v_{1} v_{1}\right), & M^{f}\left(v_{2} v_{1}\right), & \cdots, & M^{f}\left(v_{\mathbf{N}(M)} v_{1}\right) \\
M^{f}\left(v_{1} v_{2}\right), & M^{f}\left(v_{2} v_{2}\right), & \cdots, & M^{f}\left(v_{\mathbf{N}(M)} v_{2}\right) \\
\vdots & \vdots & \cdots & \vdots \\
M^{f}\left(v_{1} v_{\mathbf{N}(L)}\right), & M^{f}\left(v_{2} v_{\mathbf{N}(L)}\right), & \cdots, & M^{f}\left(v_{\mathbf{N}(M)} v_{\mathbf{N}(L)}\right)
\end{array}\right] .
$$

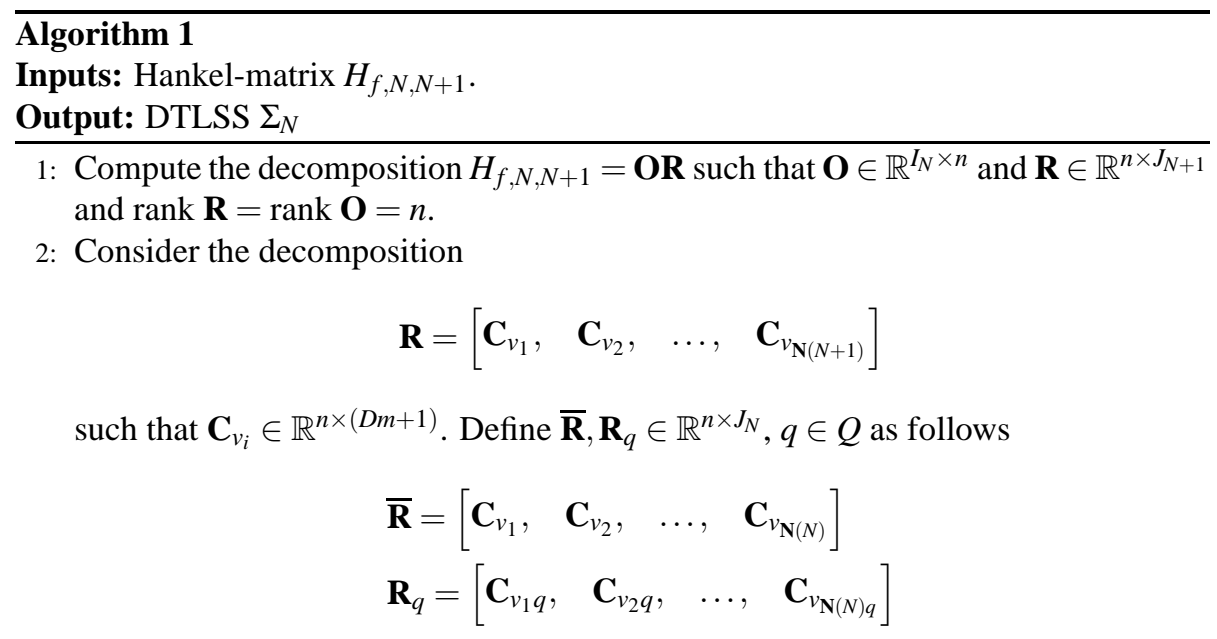

3: Construct $\Sigma_{N}$ of the form (3) such that

$$
\begin{aligned}
& {\left[x_{0}, B_{1}, \ldots, B_{D}\right]=} \\
& \text { the first } m D+1 \text { columns of } \mathbf{R} \\
& {\left[\begin{array}{lll}
C_{1}^{T}, \quad C_{2}^{T}, \quad \ldots, \quad C_{D}^{T} & { }^{T}=\text { the first } p D \text { rows of } \mathbf{O} \\
\forall q \in Q: A_{q}=\mathbf{R}_{q} \overline{\mathbf{R}}^{+},
\end{array}\right.}
\end{aligned}
$$

where $\overline{\mathbf{R}}^{+}$is the Moore-Penrose pseudoinverse of $\overline{\mathbf{R}}$.

4: Return $\Sigma_{N}$

Remark 5 (Implementation). One way to compute the factorization $H_{f, N, N+1}=\mathbf{O R}$ is as follows. If $H_{f, N, N+1}=U \Sigma V$ is the SVD decomposition of $H_{f, N, N+1}$, then define $\mathbf{O}=U \Sigma^{1 / 2}$ and $\mathbf{R}=\Sigma^{1 / 2} V$.

Theorem 6. If rank $H_{f, N, N}=\operatorname{rank} H_{f}$, then the algorithm returns a minimal realization of $f$. The condition rank $H_{f, N, N}=\operatorname{rank} H_{f}$ holds for a given $N$, if there exists an DTLSS realization $\Sigma$ of $f$ such that $\operatorname{dim} \Sigma \leq N+1$.

The proof of Theorem 6 can be found in $\$ 7$

Remark 6 (Computation of $H_{f, N, N}$ ). Note that $H_{f, N, N}$ can be computed from the responses of $f$. However, in principle, the computation of $H_{f, N, N}$ requires an exponential 
number of input/output experiments involving different switching sequences. This is clearly not very practical. It would be more practical to build $H_{f, N, N}$ based on the response of $f$ to a single switching sequence. Preliminary results on the latter approach can be found in [23]. A detailed discussion of this approach goes beyond the scope of this paper.

\section{Formal Power Series}

In this section we present an overview of the necessary results on formal power series. The material of the section is an extension of the classical theory of [5, 29], for the proofs of the results of this section see [18, 20].

Let $X$ be a finite set, which we refer to as the alphabet. A formal power series $S$ with coefficients in $\mathbb{R}^{d}$ is a map

$$
S: X^{*} \rightarrow \mathbb{R}^{d}
$$

We denote by $\mathbb{R}^{d} \ll X^{*} \gg$ the set of all such maps. Let $J$ be an arbitrary (possibly infinite) set. A family of formal power series in $\mathbb{R}^{d} \ll X^{*} \gg$ indexed by $J$, abbreviated as FFS is a collection

$$
\Psi=\left\{S_{j} \in \mathbb{R}^{d} \ll X^{*} \gg \mid j \in J\right\} .
$$

In the sequel $\Psi$ denotes a FFS of the form (14). Notice that we do not require $S_{j}, j \in J$ to be all distinct, i.e. $S_{l}=S_{j}$ for some indices $j, l \in J, j \neq l$ is allowed.

Let $J$ be an arbitrary set and let $d>0$. A $d-J$ rational representation over the alphabet $X$ is a tuple

$$
R=\left(\mathscr{X},\left\{A_{\sigma}\right\}_{\sigma \in X}, B, C\right)
$$

where $\mathscr{X}$ is a finite-dimensional vector space over $\mathbb{R}$, for each $\sigma \in X, A_{\sigma}: \mathscr{X} \rightarrow \mathscr{X}$ is a linear map, $C: \mathscr{X} \rightarrow \mathbb{R}^{d}$ is a linear map, and $B=\left\{B_{j} \in \mathscr{X} \mid j \in J\right\}$ is a family of elements of $\mathscr{X}$ indexed by $J$. If $d$ and $J$ are clear from the context we will refer to $R$ simply as a rational representation. We call $\mathscr{X}$ the state-space, $A_{\sigma}, \sigma \in X$ the state-transition maps, and $C$ the readout map of $R$. The family $B$ is called the family of initial states of $R$. The dimension $\operatorname{dim} \mathscr{X}$ of the state-space is called the dimension of $R$ and it is denoted by $\operatorname{dim} R$. If $\mathscr{X}=\mathbb{R}^{n}$, then we identify the linear maps $A_{\sigma}, \sigma \in X$ and $C$ with their matrix representations in the standard Euclidean bases, and we call them the state-transition matrices and the readout matrix respectively.

The $d-J$ representation $R$ from (15) is said to be a representation of $\Psi$, if

$$
\forall j \in J, \forall w \in X^{*}: S_{j}(w)=C A_{w} B_{j},
$$

where Notation 2 has been used. We say that the family $\Psi$ is rational, if there exists a $d-J$ representation $R$ such that $R$ is a representation of $\Psi$. A representation $R_{\min }$ of $\Psi$ is called minimal if for each representation $R$ of $\Psi, \operatorname{dim} R_{\min } \leq \operatorname{dim} R$. Define the subspaces

$$
\begin{aligned}
& W_{R}=\operatorname{Span}\left\{A_{w} B_{j} \in \mathscr{X}\left|w \in X^{*},\right| w \mid<n, j \in J\right\} \\
& O_{R}=\bigcap_{w \in X^{*},|w|<n} \operatorname{ker} C A_{w} .
\end{aligned}
$$


We will say that the representation $R$ is reachable if $\operatorname{dim} W_{R}=\operatorname{dim} R$, and we will say that $R$ is observable if $O_{R}=\{0\}$. Let $R=\left(\mathscr{X},\left\{A_{\sigma}\right\}_{\sigma \in X}, B, C\right), \widetilde{R}=\left(\widetilde{\mathscr{X}},\left\{\widetilde{A_{\sigma}}\right\}_{\sigma \in X}, \widetilde{B}, \widetilde{C}\right)$ be two $d-J$ rational representations. A linear map $\mathscr{S}: \mathscr{X} \rightarrow \widetilde{\mathscr{X}}$ is called a representation morphism, and is denoted by $\mathscr{S}: R \rightarrow \widetilde{R}$, if

$$
\mathscr{S} A_{\sigma}=\widetilde{A}_{\sigma} \mathscr{S}, \forall \sigma \in X, \quad \mathscr{S} B_{j}=\widetilde{B}_{j}, \forall j \in J, \quad C=\widetilde{C} \mathscr{S}
$$

If $\mathscr{S}$ is bijective, then it is called a representation isomorphism. If $\mathscr{S}$ is an isomorphism, then $\widetilde{R}$ and $R$ are representations of the same FFS, and $R$ is observable (reachable) if and only if $\widetilde{R}$ is observable (reachable).

Remark 7. Let $R$ be a representation of $\Psi$ of the form (15), and consider a linear isomorphism $\mathscr{S}: \mathscr{X} \rightarrow \mathbb{R}^{n}, n=\operatorname{dim} R$. Then $\mathscr{S} R=\left(\mathbb{R}^{n},\left\{\mathscr{S} A_{\sigma} \mathscr{S}^{-1}\right\}_{\sigma \in X}, \mathscr{S} B, C \mathscr{S}^{-1}\right)$, where $\mathscr{S} B=\left\{\mathscr{S} B_{j} \in \mathbb{R}^{n} \mid j \in J\right\}$ is a representation of $\Psi$ and it is isomorphic to $R$. The representation $\mathscr{S} R$ is defined on an Euclidean space and its state-transition and readout maps can be viewed as matrices.

Definition 15 (Hankel-matrix). Define the Hankel-matrix $H_{\Psi}$ of $\Psi$ as the infinite matrix, the rows of which are indexed by pairs $(v, i)$ where $v \in X^{*}, i=1, \ldots, d$, and the columns of which are indexed by $(w, j)$ where $w \in X^{*}, j \in J$. The entry $\left[H_{\Psi}\right]_{(v, i),(w, j)}$ of $H_{\Psi}$ indexed with the row index $(v, i)$ and the column index $(w, j)$ is defined as

$$
[H \Psi]_{(v, i)(w, j)}=\left[S_{j}(w v)\right]_{i}
$$

where $\left[S_{j}(w v)\right]_{i}$ denotes the ith entry of the vector $S_{j}(w v) \in \mathbb{R}^{d}$. The rank of $H_{\Psi}$ is the dimension of the linear space spanned by the columns of $H_{\Psi}$, and it is denoted by rank $H_{\Psi}$.

Theorem 7 (Existence and minimality, [18, 20]). 1. The family $\Psi$ is rational, if and only if rank $H_{\Psi}<+\infty$.

2. If rank $H \Psi<+\infty$, then a minimal representation $R$ of $\Psi$ can be constructed from $H_{\Psi}$, see Procedure 6

3. Assume that $R_{\min }$ is a representation of $\Psi$. Then $R_{\min }$ is a minimal representation of $\Psi$, if and only if $R_{\min }$ is reachable and observable. If $R_{\min }$ is minimal, then $\operatorname{rank} H_{\Psi}=\operatorname{dim} R_{\min }$.

4. All minimal representations of $\Psi$ are isomorphic.

5. Any representation $R$ of $\Psi$ can be transformed to a minimal representation $R_{\min }$ of $\Psi$, see Procedure 9

We conclude by presenting procedures for reachability and observability reduction, minimization of representations and construction of a representation from the Hankelmatrix. In the sequel, $R$ is a representation of $\Psi$ and $R$ is of the form (15).

Procedure 6 (Repr. from Hankel-matrix, [18, 20]). If rank $H_{\Psi}<+\infty$, then

$$
R_{\Psi}=\left(\operatorname{Im} H_{\Psi},\left\{A_{\sigma}\right\}_{\sigma \in X}, B, C\right)
$$


is a representation of $\Psi$. Here, for each $\sigma \in X, A_{\sigma}$ is the linear map which maps every column of $H_{\Psi}$ indexed by $(w, j)$ to the column indexed by $(w \sigma, j)$. The initial states are $B=\left\{B_{j} \mid j \in J\right\}$, where $B_{j}$ is the column of $H_{\Psi}$ indexed by $(\varepsilon, j), j \in J$. Finally, $C$ is a linear map which maps every column of $H_{\Psi}$ to the vector formed by those rows of this columns which are indexed by $(\varepsilon, 1), \ldots,(\varepsilon, d)$. Recall that $\mathbb{R}^{d}$ is set of coefficients of the formal power series $S_{j}$ of $\Psi, j \in J$, i.e. $S_{j}: X^{*} \rightarrow \mathbb{R}^{d}$.

Procedure 7 (Reachability Reduction). Assume $R$ is a representation of $\Psi$ and it is of the form (15). Recall the definition of the reachable subspace $W_{R}$ of $R$ from (17). Define the representation $R_{r}=\left(W_{R},\left\{A_{\sigma}^{r}\right\}_{\sigma \in X}, B^{r}, C^{r}\right)$, where for each $\sigma \in X, A_{\sigma}^{r}$ is the restriction of $A_{\sigma}$ to $W_{R}, B^{r}=\left\{B_{j} \in \mathscr{X} \mid j \in J\right\}=B$, and $C^{r}$ is the restriction of $C$ to $W_{R}$. Then $R_{r}$ is a reachable representation of $\Psi$.

Procedure 8 (Observability Reduction). Assume $R$ is a representation of $\Psi$ and it is of the form (15). Recall from (18) the definition of the observability subspace $O_{R}$. Define the representation $R_{o}=\left(\mathscr{X} / O_{R_{r}},\left\{\widetilde{A_{\sigma}}\right\}_{\sigma \in X}, \widetilde{B}, \widetilde{C}\right)$. Here $\mathscr{X} / O_{R}$ is the quotient space of $\mathscr{X}$ with respect to $O_{R}$. Denote by $[x], x \in \mathscr{X}$ the equivalence class of all those $y \in \mathscr{X}$ such that $x-y \in O_{R}$. Then $\widetilde{A}_{\sigma}[x]=\left[A_{\sigma} x\right], \sigma \in X, \widetilde{C}[x]=C x$ for all $x \in \mathscr{X}$, and $\widetilde{B}=\left\{\widetilde{B}_{j} \in \mathscr{X} / O_{R} \mid j \in J\right\}$ is such that $\widetilde{B}_{j}=\left[B_{j}\right], j \in J$. Then $R_{o}$ is an observable representation of $\Psi$ and if $R$ is reachable, then so is $R_{o}$.

Procedure 9 (Minimization). A representation $R$ of $\Psi$ can be converted to a minimal representation as follows. Use Procedure 7 to obtain a reachable representation $R_{r}$. Apply Procedure 8 to $R_{r}$ and obtain the observable representation $R_{\min }=\left(R_{r}\right)_{o}$. Then $R_{\min }$ is a minimal representation of $\Psi$.

If $J$ is finite, then Procedures 6, 7, 8, and 9 can be implemented, see [18].

More precisely, we can formulate a realization algorithm for rational representations, [24]. Below we present slight extension of the results of [28, 29, 12] on realization algorithms for formal power series. The proofs of the results can be found in [18, 24]. We introduce the following notation. Let $K, M \in \mathbb{N}$.

$$
\begin{aligned}
& \mathbb{I}_{M}=\left\{(v, i)\left|v \in X^{*},\right| v \mid \leq M, i=1, \ldots, p\right\} \\
& \mathbb{J}_{K}=\left\{(w, j)\left|j \in J, w \in X^{*},\right| w \mid \leq K\right\}
\end{aligned}
$$

Intuitively, the elements of $\mathbb{I}_{M}$ (resp. $\mathbb{J}_{K}$ ) are those row (column) indices of $H_{\Psi}$, the $X^{*}$-valued component of which is of length at most $M$ (resp. $K$ ).

Definition 16. Define the matrix $H_{\Psi, M, K}$ as the matrix, rows of which are indexed by the elements of $\mathbb{I}_{M}$, columns of which are indexed by the elements of $\mathbb{J}_{K}$, and its entry $\left(H_{\Psi, M, K}\right)_{(v, i),(w, j)}$ indexed by the row index $(v, i) \in \mathbb{I}_{M}$ and the column index $(w, j) \in$ $\mathbb{J}_{K}$ is defined as $\left(H_{\Psi, M, K}\right)_{(v, i),(w, j)}=\left(H_{\Psi}\right)_{(v, i),(w, j)}=\left(S_{j}(w v)\right)_{i}$. The rank of $H_{\Psi, M, K}$, denoted by rank $H_{\Psi, M, K}$, is the dimension of the linear space spanned by its columns.

That is, $H_{\Psi, M, K}$ is the sub-matrix of $H_{\Psi}$ formed by the intersection of the columns indexed by the elements of $\mathbb{J}_{K}$ and of the rows indexed by the elements of $\mathbb{I}_{M}$. If $J$ is finite, then $H_{\Psi, M, K}$ is a finite matrix. 
Theorem 8 (Realization algorithm, [24]). If rank $H_{\Psi, N, N}=\operatorname{rank} H_{\Psi}$, then the representation $R_{N}$, to be defined below, is a minimal representation of $\Psi$. If $\operatorname{rank} H_{\Psi} \leq N+1$, then $\operatorname{rank} H_{\Psi, N, N}=\operatorname{rank} H_{\Psi}$ holds. The representation $R_{N}$ is of the form (15), with the state-space $\mathscr{X}=\operatorname{Im} H_{\Psi, N, N+1}$, and such that if we denote by $\mathbf{C}_{w, j}$ the column of $\operatorname{Im} H_{\Psi, N, N}$ indexed by $(w, j) \in \mathbb{J}_{N}$, then

$$
\begin{aligned}
& \forall(w, j) \in \mathbb{J}_{N}: A_{\sigma}\left(\mathbf{C}_{w, j}\right)=\mathbf{C}_{w \sigma, j} \\
& \forall(w, j) \in \mathbb{J}_{N}: C\left(\mathbf{C}_{w, j}\right)=\left[\mathbf{C}_{w, j}((\varepsilon, 1)), \quad \cdots, \quad \mathbf{C}_{w, j}((\varepsilon, p))\right]^{T} \\
& \forall j \in J: B_{j}=\mathbf{C}_{\varepsilon, j}
\end{aligned}
$$

Here $C_{w, j}((\varepsilon, i))$ is the entry of the column $\mathbf{C}_{w, j}$ indexed by $(\varepsilon, i)$. i.e. it equals $\left(H_{\Psi, N, N}\right)_{(\varepsilon, i),(w, j)}, i=1, \ldots, p$.

\section{Proof of the main results}

The proof of the results on realization theory relies on the relationship between formal power series representations and DTLSSs state-space representations. This relationship is completely analogous to the one for linear switched systems in continuous time, [20, 19].

Consider an input-output map $f$ and assume that $f$ has a GCR. Below we define the FFS $\Psi_{f}$ associated with $f$. We also define the representation $R_{\Sigma}$ associated with a DTLSS $\Sigma$ and a DTLSS $\Sigma_{R}$ associated with a rational representation $R$. These notions allow us to relate FFS and input-output maps and to relate DTLSS with rational representations. In turn, these correspondences enable us to translate the realization problem for DTLSS to the problem of rationality of FFS.

We first define the FFS associated with $f$. To this end, recall the definition (8) of the Markov-parameters of $f$.

Definition 17 (FFS associated with $f$ ). For each $q \in Q$, each index $j=1, \ldots, m$, define the formal power series $\mathbb{S}_{q, j}, \mathbb{S}_{0} \in \mathbb{R}^{p D} \ll Q^{*} \gg$ as follows; for each word $w \in Q^{*}$, discrete mode $q \in Q$ and index $j=1, \ldots, m$,

$$
\begin{aligned}
& \mathbb{S}_{(q, j)}(w)=\left[\begin{array}{llll}
\left(S_{j}^{f}(q w 1)\right)^{T}, & \left(S_{j}^{f}(q w 2)\right)^{T}, & \cdots, & \left(S_{j}^{f}(q w D)\right)^{T}
\end{array}\right]^{T}, \\
& \mathbb{S}_{0}(w)=\left[\begin{array}{llll}
\left(S_{0}^{f}(w 1)\right)^{T}, & \left(S_{0}^{f}(w 2)\right)^{T}, & \cdots, & \left(S_{0}^{f}(w D)\right)^{T}
\end{array}\right]^{T} .
\end{aligned}
$$

Let $J_{f}=\{0\} \cup\{(q, l) \mid q \in Q, l=1, \ldots, m\}$ and define the FFS associated with $f$ by

$$
\Psi_{f}=\left\{\mathbb{S}_{j} \in \mathbb{R}^{p D} \ll Q^{*} \gg \mid j \in J_{f}\right\} .
$$

Notice that the values of $\mathbb{S}_{(q, j)}(w)$ and $S_{0}^{f}(w)$ are obtained by stacking up the Markov-parameters of $S_{j}^{f}(q w i)$ and $S_{0}^{f}(w i)$ respectively, for $i=1, \ldots, D$. Next, we define the representation $R_{\Sigma}$ associated with $\Sigma$.

Definition 18. Assume that $\Sigma$ is of the form (3). Define the representation $R_{\Sigma}$ associated with $\Sigma$ as a $p|Q|-J_{f}$ representation of the form (15), where $J_{f}=\{0\} \cup Q \times\{1, \ldots, m\}$ and the following holds. 
- The alphabet $X$ of $R_{\Sigma}$ is the set of discrete modes $Q$, and $d=p|Q|$.

- The state-space $\mathscr{X}$ of $R_{\Sigma}$ is the same as that of $\Sigma$, i.e. $\mathscr{X}=\mathbb{R}^{n}$. For each $q \in Q$, the state-transition matrix $A_{q}$ of $R_{\Sigma}$ is identical to the matrix $A_{q}$ of $\Sigma$.

- The $p|Q| \times n$ readout matrix $C$ is obtained by vertically "stacking up" the matrices $C_{1}, \ldots, C_{D}$, i.e.

$$
C=\left[\begin{array}{llll}
C_{1}^{T}, & C_{2}^{T}, & \cdots & C_{D}^{T}
\end{array}\right]^{T} \in \mathbb{R}^{p D \times n} .
$$

- $B=\left\{B_{j} \in \mathscr{X} \mid j \in J_{f}\right\}$, where $B_{0}=x_{0}$ and $B_{(q, l)}$ is the lth column of the matrix $B_{q}$ of $\Sigma$.

The intuition behind the definition of $R_{\Sigma}$ is that we would like $R_{\Sigma}$ to be a representation of $\Psi_{f}$ if and only if (24) holds. Then the $A_{q}$ matrices of the representation $R_{\Sigma}$ should coincide with the $A_{q}$ matrices of $\Sigma$. The initial states of $R_{\Sigma}$ should be formed by the vector $B_{0}$ (in order to generate $\mathbb{S}_{0}$ ), and $B_{q} e_{j}$ (in order to generate $\mathbb{S}_{(q, j)}$ ). Finally, the readout map $C$ should be formed by "stacking up" the matrices $C_{q}$. Next, we define a DTLSS $\Sigma_{R}$ based on a representation $R$.

Definition 19. Consider a $p|Q|-J_{f}$ representation $R$ of the form $(15)$, over the alphabet $X=Q$ with $d=p|Q|$. If $\mathscr{X}=\mathbb{R}^{n}$ does not hold, then replace $R$ with the isomorphic copy $\mathscr{S} R$ defined in Remark 7 whose state-space is $\mathbb{R}^{n}$. In the rest of the construction, we assume that $\mathscr{X}=\mathbb{R}^{n}$ for $n=\operatorname{dim} \mathscr{X}$ holds and that $A_{q}, q \in Q$ are $n \times n$ matrices, and $C$ is a $p|Q| \times n$ matrix. Define the DTLSS $\Sigma_{R}$ associated with R as follows. Let $\Sigma_{R}$ be of the form (3) such that

- for $q \in Q$, the matrix $A_{q}$ of $\Sigma_{R}$ is identical to the state-transition matrix $A_{q}$ of $R$.

- For each $q \in Q$, the matrix $C_{q}$ is formed by the rows $(q-1) p+1,(q-1) p+$ $2, \ldots, q p$ of $C$, i.e.

$$
C=\left[\begin{array}{llll}
C_{1}^{T}, & C_{2}^{T}, & \cdots & C_{D}^{T}
\end{array}\right]^{T} .
$$

- For each $q \in Q, B_{q}=\left[B_{(q, 1)}, \quad \cdots \quad B_{(q, m)}\right]$. The initial state $x_{0}$ of $\Sigma_{R}$ is defined as $x_{0}=B_{0}$.

The intuition behind the definition of $\Sigma_{R}$ is the following. We would like $\Sigma_{R}$ to be such that if we apply Definition 18 to it, then the resulting representation $R_{\Sigma_{R}}$ should be close to $R$.

The relationship between the various concepts introduced above is as follows.

Theorem 9. 1. The Hankel-matrix $H_{\Psi_{f}}$ equals the Hankel-matrix $H_{f}$ of $f$.

2. The representations $R$ and $R_{\Sigma_{R}}$ are isomorphic, and $\Sigma_{R_{\Sigma}}=\Sigma$.

3. The DTLSS $\Sigma$ is a realization of the input-output map $f$ if and only if the associated representation $R_{\Sigma}$ is a representation of $\Psi_{f}$.

4. The representation $R$ is a representation of $\Psi_{f}$ if and only if the associated DTLSS $\Sigma_{R}$ is a realization of $f$. 
5. The DTLSS $\Sigma$ is a minimal realization of the input-output map $f$ if and only if the associated representation $R_{\Sigma}$ is a minimal representation of $\Psi_{f}$.

6. The representation $R$ is a minimal representation of $\Psi_{f}$ if and only if the associated DTLSS $\Sigma_{R}$ is a minimal realization of $f$.

7. The DTLSS $\Sigma$ is span-reachable (observable) if and only if the associated representation $R_{\Sigma}$ is reachable (resp. observable).

8. The representation $R$ is reachable (observable) if and only if the associated DTLSS $\Sigma_{R}$ is span-reachable (resp. observable).

9. Assume that $\Sigma_{1}$ and $\Sigma_{2}$ are two DTLSSs with the state-spaces $\mathbb{R}^{n}$ and $\mathbb{R}^{n_{a}}$ respectively. A matrix $\mathscr{S} \in \mathbb{R}^{n_{a} \times n}$ is a DTLSS morphism $\mathscr{S}: \Sigma_{1} \rightarrow \Sigma_{2}$ if and only if $\mathscr{S}: R_{\Sigma_{1}} \rightarrow R_{\Sigma_{2}}$ is a representation morphism, if $\mathscr{S}$ is interpreted as a linear map.

The statements of Theorem 9 above are summarized in Table 1

Proof of 9 Proof of Part 1 , Straightforward.

Proof of Part2, Straightforward.

Proof of Part 3 and Part 4. The proof is analogous to the proof of Theorem 10 from [20]. First, note that if $R$ is a representation of $\Psi_{f}$, then $R$ satisfies the assumptions of Definition 19 Since $R$ is isomorphic to $R_{\Sigma_{R}}$, Part 4 follows from Part 3 , Part 3 follows by noticing that $\Sigma$ is a realization of $f$, if and only if for all $q_{0} \in Q, j=1, \ldots, m$, $w \in Q^{*}$,

$$
\begin{aligned}
& \mathbb{S}_{\left(q_{0}, j\right)}(w)=\left[\begin{array}{llll}
C_{1}^{T}, & C_{2}^{T}, & \cdots, & C_{D}^{T}
\end{array}\right]^{T} A_{w} B_{q_{0}} e_{j} \text { and } \\
& \mathbb{S}_{0}(w)=\left[\begin{array}{llll}
C_{1}^{T}, & C_{2}^{T}, & \cdots, & C_{D}^{T}
\end{array}\right]^{T} A_{w} x_{0} .
\end{aligned}
$$

The above statement follows from Lemma 1 by taking into account the definition of $\mathbb{S}_{0}$ and $\mathbb{S}_{\left(q_{0}, j\right)}$. But $(24)$ is equivalent to $R_{\Sigma}$ being a representation of $\Psi_{f}$. Indeed, the matrix $\left[\begin{array}{llll}C_{1}^{T} & C_{2}^{T}, & \cdots & C_{D}^{T}\end{array}\right]^{T}$ in the right-hand side of (24) equals the readout matrix $C$ of $R_{\Sigma}$, and the vectors $B_{q_{0}} e_{j}$ and $x_{0}$ coincide with the initial states $B_{\left(q_{0}, j\right)}$ and $B_{0}$ of $R_{\Sigma}$. Hence, (24) in fact says that $\mathbb{S}_{j}(w)=C A_{w} B_{j}$ for all $w \in Q^{*}, j \in J_{f}$, i.e. that $R_{\Sigma}$ is a representation of $\Psi_{f}$.

Proof of Part 5 and Part 6. Follows from Part 3 and Part 4, by noticing that $\operatorname{dim} \Sigma=\operatorname{dim} R_{\Sigma}$ and $\operatorname{dim} R=\operatorname{dim} \Sigma_{R}$.

Proof of Part 7 and 8. Since $R_{\Sigma_{R}}$ is isomorphic to $R$, it is enough to prove Part 77 To that end it is enough to show that $W_{R_{\Sigma}}=\operatorname{Im} R(\Sigma)$ and $O_{R_{\Sigma}}=\operatorname{ker} O(\Sigma)$, i.e. the image of the reachability matrix of $\Sigma$ equals the space $W_{R_{\Sigma}}$ of $R_{\Sigma}$, and the kernel of the observability matrix of $\Sigma$ equals $O_{R_{\Sigma}}$.

Assume that $R_{\Sigma}$ is of the form (15), with $\mathscr{X}=\mathbb{R}^{n}, d=p|Q|$ and $X=Q$. To see that $\operatorname{Im} R(\Sigma)=W_{R_{\Sigma}}$, notice that $\operatorname{Im} \mathscr{R}(\Sigma)$ is the linear span of the columns of matrices $A_{w} B_{q}$ and vectors $A_{w} x_{0}, q \in Q, w \in Q^{*},|w|<n$. But the initial states $B$ of $R_{\Sigma}$ consists of the columns of the matrices $B_{q}, q \in Q$, and of the vector $x_{0}$. Hence, $\operatorname{Im} \mathscr{R}(\Sigma)$ is spanned by vectors $A_{w} B_{j}, j \in J_{f}$ and hence it equals $W_{R_{\Sigma}}$. 
Similarly, the kernel of $O(\Sigma)$ equals the intersection of $\operatorname{ker} C_{q} A_{w}, q \in Q, w \in Q^{*}$, $|w|<n$. It is easy to see that $\bigcap_{q \in Q} \operatorname{ker} C_{q} A_{w}=\operatorname{ker} C A_{w}$, hence, $\operatorname{ker} O(\Sigma)$ is the intersection of all spaces $\operatorname{ker} C A_{w}, w \in Q^{*},|w|<n$. But the latter intersection equals $O_{R_{\Sigma}}$.

Proof of Part 9 . The proof is analogous to the proof of Lemma 10 of [20]. Since the state-spaces of $R_{\Sigma_{1}}$ and $\Sigma_{1}$ are the same, and the state-spaces of $R_{\Sigma_{2}}$ and $\Sigma_{2}$ are the same, $\mathscr{S}$ can indeed be viewed both as a potential representation morphism from $R_{\Sigma_{1}}$ to $R_{\Sigma_{2}}$ and as a potential DTLSS morphism from $\Sigma_{1}$ to $\Sigma_{2}$. Then it is enough to prove that $\mathscr{S}$ satisfies (19) with $R=R_{\Sigma_{1}}$ and $\widetilde{R}=R_{\Sigma_{2}}$ if and only if $\mathscr{S}$ satisfies Definition 9 . The latter proof is routine. Indeed, assume that $\Sigma_{1}$ is of the form (3) and that $\Sigma_{2}$ is of the form

$$
\Sigma_{2}=\left(n^{\prime}, Q,\left\{\left(A_{q}^{\prime}, B_{q}^{\prime}, C_{q}^{\prime}\right) \mid q \in Q\right\}, x_{0}^{\prime}\right) .
$$

Assume that $R_{\Sigma_{1}}$ is of the form (15) and $R_{\Sigma_{2}}=\left(\mathbb{R}^{\prime},\left\{A_{q}^{\prime}\right\}_{q \in Q}, B^{\prime}, C^{\prime}\right)$ where $B^{\prime}=\left\{B_{j}^{\prime} \mid\right.$ $\left.j \in J_{f}\right\}$. Note that the matrices $A_{q}$ and $A_{q}^{\prime}$ of $R_{\Sigma_{1}}$, respectively $R_{\Sigma_{2}}$, coincide with the corresponding matrices of $\Sigma_{1}$ and $\Sigma_{2}$. Then $\mathscr{S}$ is a DTLSS morphism if and only if

$$
\left(\forall q \in Q: \mathscr{S} A_{q}=A_{q}^{\prime} \mathscr{S}, C_{q}=C_{q}^{\prime} \mathscr{S}, \mathscr{S} B_{q}=B_{q}^{\prime}\right)
$$

$$
\text { and } \mathscr{S} x_{0}=x_{0}^{\prime} \text {. }
$$

But $\forall q \in Q: C_{q}=C_{q}^{\prime} \mathscr{S}$ is equivalent to $C=C^{\prime} \mathscr{S}$, since

$$
\begin{aligned}
C & =\left[\begin{array}{lll}
\left(C_{1}\right)^{T}, & \cdots, & \left(C_{D}\right)^{T}
\end{array}\right]^{T} \\
& =\left[\begin{array}{lll}
\left(C_{1}^{\prime} \mathscr{S}\right)^{T}, & \cdots, & \left(C_{D}^{\prime} \mathscr{S}\right)^{T}
\end{array}\right]^{T}=C^{\prime} \mathscr{S} .
\end{aligned}
$$

Similarly, $\mathscr{S} B_{q}=B_{q}^{\prime}$ is equivalent to: $\forall l=1, \ldots, m, \mathscr{S} B_{q} e_{l}=\mathscr{S} B_{(q, l)}=B_{q}^{\prime} e_{l}=B_{(q, l)}^{\prime}$. This, together with $\mathscr{S} x_{0}=x_{0}^{\prime}$, implies that $\mathscr{S} B_{j}=B_{j}^{\prime}$ for all $j \in J_{f}$.

Hence, we have established that $\mathscr{S}$ is a DTLSS morphism if and only if $\forall q \in Q$ : $\mathscr{S} A_{q}=A_{q}^{\prime} \mathscr{S}, C=C^{\prime} \mathscr{S}$, and $\forall j \in J_{f}: \mathscr{S} B_{j}=B_{j}^{\prime}$. But this means that $\mathscr{S}: R_{\Sigma_{1}} \rightarrow R_{\Sigma_{2}}$ is a representation morphism.

Proof Theorem 3 By Theorem 9, Part5, $\Sigma$ is a minimal DTLSS realization of $f$ if and only if $R=R_{\Sigma}$ is minimal. By Theorem $7, R$ is minimal if and only if $R$ is reachable and observable. By Theorem 9 , Part 7 , the latter is equivalent to $\Sigma$ being span-reachable and observable. Next, we show that minimal DTLSS realizations of $f$ are isomorphic. Let $\Sigma$ and $\hat{\Sigma}$ be two minimal DTLSS realizations of $f$. By Theorem 9 Part $5, R_{\Sigma}$ and $R_{\hat{\Sigma}}$ are minimal representations of $\Psi_{f}$. Then from Theorem 7 it follows that there exists a isomorphism $\mathscr{S}: R_{\hat{\Sigma}} \rightarrow R_{\Sigma}$. From Part 9 of Theorem 9 is then follows that $\mathscr{S}: \hat{\Sigma} \rightarrow \Sigma$ is an isomorphism. Finally, the correctness of Procedure 4 is shown in Remark 10 .

\section{Proof of Theorem 5 Necessity}

Assume that $\Sigma$ is a DTLSS which is a realization of $f$. Then by Lemma $1, f$ has a GCR. Moreover, by Theorem $9, R_{\Sigma}$ is a representation of $\Psi_{f}$, i.e. $\Psi_{f}$ is rational. By Theorem 9 Part1 and Theorem[7 the latter implies that rank $H_{f}<+\infty$. 


\section{Sufficiency}

Assume that $f$ has a GCR and rank $H_{f}<+\infty$. Then by Theorem 9, Part 1 and Theo$\operatorname{rem} 7 \Psi_{f}$ is rational, i.e. it has a representation $R$. Then by Theorem 9 the DTLSS $\Sigma_{R}$ is a realization of $f$, i.e. $f$ has a realization.

Finally, the correctness of Procedure 5 follows from Remark 11 below.

Now we are ready to analyze Procedure $2,3,4$ and 5.

Remark 8 (Correctness of Procedure 2). Procedure 2 is equivalent to the following procedure. Apply Procedure 7 to $R_{\Sigma}$ to obtain $R_{r}$. Then $\Sigma_{r}$ from Procedure 2 and $\Sigma_{R_{r}}$ are isomorphic. It then follows that $\Sigma_{r}$ is span-reachable, since $R_{r}$ is reachable, and $\Sigma_{r}$ and $\Sigma$ have the same input-output map, since both $R_{\Sigma}$ and $R_{r}$ are representations of $\Psi_{y_{\Sigma}}$.

Remark 9 (Correctness of Procedure 3). Procedure 3 is equivalent to the following procedure. Apply Procedure 8 to $R_{\Sigma}$ to obtain an observable representation $R_{o}$. It follows that $\Sigma_{o}$ from Procedure 3 and $\Sigma_{R_{o}}$ are isomorphic. Since $R_{o}$ is observable, $\Sigma_{o}$ is observable as well. If $\Sigma$ is span-reachable, then $R_{\Sigma}$ is reachable. Hence, then $R_{o}$ is reachable and thus $\Sigma_{o}$ is span-reachable. Finally, both $R_{\Sigma}$ and $R_{o}$ are representations of $\Psi_{y_{\Sigma}}$, from which it follows that the input-output maps of $\Sigma$ and $\Sigma_{o}$ coincide.

Remark 10 (Correctness of Procedure 4). Procedure 4 can be restated as follows. Apply Procedure 9 to $R_{\Sigma}$ and denote the resulting minimal representation by $R_{m}$. It then follows that $\Sigma_{m}$ from Procedure 4 is isomorphic to $\Sigma_{R_{m}}$. Since by Theorem $9 \Sigma_{R_{m}}$ is a minimal realization of $y_{\Sigma}$, then so is $\Sigma_{m}$.

Remark 11 (Correctness of Procedure 5). Procedure 5 can be reformulated as follows. Use Procedure 6 to construct a minimal representation $R$ of $\Psi_{f}$ from $H_{f}=H_{\Psi_{f}}$. Then by Theorem $9 \Sigma_{R}$ will be a minimal realization of $f$. It is easy to see that the DTLSS $\Sigma_{f}$ from Procedure 5 is isomorphic to $\Sigma_{R}$.

We will continue with the proof of Theorem 6

Proof of Theorem 6 The proof is almost the same as that of the continuous-time case, described in [25]. From Theorem 9 it follows that $H_{f, K, L}$ coincides with $H_{\Psi_{f}, K, L}$, and hence, rank $H_{f, N, N}=\operatorname{rank} H_{f}$ is equivalent to $\operatorname{rank} H_{\Psi_{f}, N, N}=\operatorname{rank} H_{\Psi_{f}}$.

Assume now that rank $H_{f, N, N}=\operatorname{rank} H_{f}$. Then the representation $R_{N}$ from Theorem 8 is well-defined and it is a minimal representation of $\Psi_{f}$. Consider Algorithm 1 and the decomposition defined there. Then $\operatorname{Im} H_{f, N, N+1}=\operatorname{ImO}$ and there exists a left inverse $\mathbf{O}^{+} \in \mathbb{R}^{n \times I_{N}}$ of $\mathbf{O}$ such that $\mathbf{O}^{+} \mathbf{O}=I_{n}$.

Consider the linear map $\mathscr{S}: \operatorname{Im} H_{f, N, N+1} \rightarrow \mathbb{R}^{n}$, where $\mathscr{S}(x)=\mathbf{O}^{+} x$ for all $x \in$ $\operatorname{Im} H_{f, N, N+1}$ and recall that $H_{f, N, N+1}=H_{\Psi_{f}, N, N+1}$. It then follows that $\mathscr{S}$ is a linear isomorphism, and its inverse is $\mathbf{O}$. Moreover, the isomorphic copy

$$
\mathscr{S} R_{N}=\left(\mathbb{R}^{n},\left\{\mathscr{S} A_{q} \mathscr{S}^{-1}\right\}_{q \in Q},\left\{\mathscr{S}\left(B_{j}\right) \mid j \in J_{f}\right\}, C \mathscr{S}^{-1}\right)
$$

of $R_{N}$ is also a minimal representation of $\Psi_{f}$.

Consider now the DTLSS $\Sigma_{\mathscr{S} R_{N}}$ associated with $\mathscr{S} R_{N}$. It is easy to see that the DTLSS $\Sigma_{\mathscr{S} R_{N}}$ satisfies 11, 13 and hence it coincides with the DTLSS $\Sigma_{N}$ returned by Algorithm 1. Theorem 9 it follows then that $\Sigma_{N}$ is a minimal realization of $f$. 
Assume that there exists an DTLSS realization $\Sigma$ of $f$, such that $\operatorname{dim} \Sigma \leq N+1$. Then by Theorem 5, $\operatorname{rank} H_{f}=\operatorname{rank} H_{\Psi_{f}} \leq \operatorname{dim} \Sigma \leq N+1$. Hence, by Theorem 8 , $\operatorname{rank} H_{\Psi_{f}}=\operatorname{rank} H_{\Psi_{f}, N, N}$.

We conclude this section with the following remark.

Remark 12 (Continuous-time case). If instead of a discrete-time system we consider a continuous-time system $\Sigma$, then the constructions of $R_{\Sigma}$ and $\Sigma_{R}$ are exactly the same. The construction of $\Psi_{f}$ differs only in the way the Markov-parameters $S_{j}^{f}\left(q_{0} v q\right)$ and $S_{0}^{f}(v q), v \in Q^{*}, q, q_{0} \in Q, j=1, \ldots, m$, are derived from the input-output map $f$. However, $S_{0}^{f}(v q)=C_{q} A_{v} x_{0}$ and $S_{j}^{f}\left(q_{0} v q\right)=C_{q} A_{v} B_{q_{0}} e_{j}$ also holds for the continuous-time case, if $\Sigma$ is a realization of $f$. A detailed description of the continuous-time case can be found in [20, 19].

\begin{tabular}{|cccc|}
\hline Realization of $f$ & & Representation of $\Psi_{f}$ \\
\hline$\Sigma=\Sigma_{R_{\Sigma}}$ & $\Longleftrightarrow$ & $R_{\Sigma}$ \\
$\Sigma_{R}$ & $\Longleftrightarrow$ & $R=R_{\Sigma_{R}}$ \\
observable, span-reachable & $\Longleftrightarrow$ & observable, reachable \\
minimal & $\Longleftrightarrow$ & minimal \\
$\mathscr{S}$, DTLSSmorphism & $\Longleftrightarrow$ & $\mathscr{S}$, representation morphism \\
\hline
\end{tabular}

Table 1: Correspondence between DTLSSs and representations

\section{Conclusions}

We presented realization theory for discrete-time linear switched systems. The results and the proof techniques resemble the ones for continuous-time linear switched systems presented in our previous work.

\section{References}

[1] L. Bako, G. Merc Ã “re, and S. Lecoeuche. Online structured subspace identification with application to switched linear systems. International Journal of Control, 82:1496-1515, 2009.

[2] L. Bako, G. Merc Ã re, R. Vidal, and S. Lecoeuche. Identification of switched linear state space models without minimum dwell time. In IFAC Symposium on System Identification, Saint Malo, France, 2009.

[3] J.A. Ball, G. Groenewald, and T. Malakorn. Structured noncommutative multidimensional linear systems. SIAM J. on Control and Optimization, 44(4):14741528, 2005. 
[4] A. Bemporad, A. Garulli, S. Paoletti, and A. Vicino. A bounded-error approach to piecewise affine system identification. IEEE Transactions on Automatic Control, 50(10):1567-1580, 2005.

[5] J. Berstel and C. Reutenauer. Rational series and Their Languages. SpringerVerlag, 1984.

[6] P. Collins and J. H. van Schuppen. Observability of piecewise-affine hybrid systems. In Hybrid Systems: Computation and Control, 2004.

[7] Samuel Eilenberg. Automata, Languages and Machines. Academic Press, New York, London, 1974.

[8] G. Ferrari-Trecate, M. Muselli, D. Liberati, and M. Morari. A clustering technique for the identification of piecewise affine systems. Automatica, 39:205-217, 2003.

[9] M. Fliess. Matrices de hankel. J. Math. Pures Appl., (23):197 - 224, 1973.

[10] F. Gécseg and I Peák. Algebraic theory of automata. Akadémiai Kiadó, Budapest, 1972.

[11] R.L. Grossman and R.G. Larson. An algebraic approach to hybrid systems. Theoretical Computer Science, 138:101-112, 1995.

[12] A. Isidori. Direct construction of minimal bilinear realizations from nonlinear input-output maps. IEEE Transactions on Automatic Control, pages 626-631, 1973.

[13] Nathan Jacobson. Lectures in Abstract Algebra, volume II: linear algebra. D. van Nostrand Company, Inc. New York, 1953.

[14] A. Lj. Juloski, S. Weiland, and W.P.M.H. Heemels. A bayesian approach to identification of hybrid systems. IEEE Transactions on Automatic Control, 50:15201533, 2005.

[15] H. Nakada, K. Takaba, and T. Katayama. Identification of piecewise affine systems based on statistical clustering technique. Automatica, 41:905-913, 2005.

[16] S. Paoletti, A. Juloski, G. Ferrari-Trecate, and R. Vidal. Identification of hybrid systems: A tutorial. European Journal of Control, 13(2-3):242 - 260, 2007.

[17] S. Paoletti, J. Roll, A. Garulli, and A. Vicino. Input/ouput realization of piecewise affine state space models. In 46th IEEE Conf. on Dec. and Control, 2007.

[18] M. Petreczky. Realization Theory of Hybrid Systems. PhD thesis, Vrije Universiteit, Amsterdam, 2006.

[19] M. Petreczky. Realization theory for linear switched systems: Formal power series approach. Systems \& Control Letters, 56:588-595, 2007. 
[20] M. Petreczky. Realization theory of linear and bilinear switched systems: A formal power series approach: Part i. ESAIM Control, Optimization and Calculus of Variations, 2010. DOI 10.1051/cocv/2010014.

[21] M. Petreczky, L. Bako, and J.H. van Schuppen. Identifiability of discrete-time linear switched systems. In Hybrid Systems: Computation and Control. ACM, 2010.

[22] M. Petreczky and J.H. van Schuppen. Realization theory for linear hybrid systems. IEEE Trans. on Automatic Control, 55(10):2282 - 2297, 2010.

[23] Mihaly Petreczky and Laurent Bako. On the notion of persistence of excitation for linear switched systems. In 50th IEEE Conference on Decision and Control, 2011.

[24] Mihaly Petreczky and Jan H. van Schuppen. Partial-realization of linear switched systems: A formal power series approach. Technical Report arXiv:1010.5160v1, ArXiv, 2010. Available at http://arxiv.org/abs/1010.5160v1

[25] Mihaly Petreczky and Jan H. van Schuppen. Partial-realization of linear switched systems: A formal power series approach. Automatica, 47:1277-2184, 2011.

[26] Mihaly Petreczky and René Vidal. Realization theory for semi-algebraic hybrid systems. In Hybrid Systems: Computation and Control, pages 386-400, 2008.

[27] J. Roll, A. Bemporad, and L. Ljung. Identification of piecewise affine systems via mixed-integer programming. Automatica, 40:37-50, 2004.

[28] Eduardo D. Sontag. Polynomial Response Maps, volume 13 of Lecture Notes in Control and Information Sciences. Springer Verlag, 1979.

[29] Eduardo D. Sontag. Realization theory of discrete-time nonlinear systems: Part I - the bounded case. IEEE Trans. on Circuits and Systems, CAS-26(4), 1979.

[30] Zhendong Sun and Shuzhi S. Ge. Switched linear systems : control and design. Springer, London, 2005.

[31] J.M. van den Hof. System theory and system identification of compartmental systems. PhD thesis, University of Groningen, 1996.

[32] V. Verdult and M. Verhaegen. Subspace identification of piecewise linear systems. In Proc.Conf. Decision and Control, 2004.

[33] R. Vidal. Recursive identification of switched ARX systems. Automatica, 44(9):2274 - 2287, 2008.

[34] R. Vidal, A. Chiuso, and S. Sastry. Observability and identifiability of jump linear systems. In Proc. IEEE Conf. Dec. and Control, pages 3614 - 3619, 2002.

[35] S. Weiland, A. Lj. Juloski, and B. Vet. On the equivalence of switched affine models and switched ARX models. In 45th IEEE Conf. on Decision and Control, 2006. 


\section{A Technical proofs}

Proof of Lemma 1 Consider the input-output map $y_{\Sigma}$ of $\Sigma$. By induction on $t$, it follows that if $w=(v, u) \in \mathscr{U}^{+}, v=q_{0} \cdots q_{t}, u=u_{0} \cdots u_{t}, t \geq 0, q_{0}, \ldots, q_{t} \in Q, u_{0}, \ldots, u_{t} \in$ $\mathbb{R}^{m}$, then

$$
y_{\Sigma}(w)=C_{q_{t}} A_{v_{0 \mid t-1}} x_{0}+\sum_{j=0}^{t-1} C_{q_{t}} A_{v_{j+1 \mid t-1}} B_{q_{j}} u_{j}
$$

Consider the Markov-parameters $S_{0}^{y_{\Sigma}}(s q), S_{j}^{y_{\Sigma}}\left(q_{0} s q\right), q, q_{0} \in Q, s \in Q^{*}, j=1, \ldots, m$, of $y_{\Sigma}$. It then follows from (25) and the definition of Markov-parameters that for all $s \in Q^{*}$,

$$
S_{0}^{y_{\Sigma}}(s q)=C_{q} A_{s} x_{0} \text { and } S_{j}^{y_{\Sigma}}\left(q_{0} s q\right)=C_{q} A_{s} B_{q_{0}} e_{j} .
$$

Notice that (25) - (26) implies that $y_{\Sigma}$ has a generalized convolution representation.

Assume that $\Sigma$ is a realization of $f$. Then $y_{\Sigma}=f$. Then from (25)-(26) it follows that $f$ has a generalized convolution representation and (9) holds. Conversely, assume that $f$ has a generalized convolution representation and that (9) holds. From (9) it follows that the Markov-parameters of $y_{\Sigma}$ and $f$ coincide, i.e. $S_{0}^{y_{\Sigma}}(s q)=S_{0}^{f}(s q)$ and $S_{j}^{y_{\Sigma}}\left(q_{0} s q\right)=S_{j}^{f}\left(q_{0} s q\right)$ for all $s \in Q^{*}, q, q_{0} \in Q, j=1, \ldots, m$. Since both $y_{\Sigma}$ and $f$ admit a generalized convolution representation, by Remark 2 they are equal. The latter means that $\Sigma$ is a realization of $f$.

Proof of Theorem 4 It is enough to show that for any family of $n \times n$ matrices $F_{q}$, $q \in Q$ and any matrix $G \in \mathbb{R}^{n \times l}$ for some $l>0$ the following holds. Define the matrix $\mathscr{R}_{k}=\left[\begin{array}{lll}F_{v_{1}} G & \ldots & F_{v_{M_{k+1}}} G\end{array}\right]$ for $k \in \mathbb{N}$. That is, $\mathscr{R}_{k}$ is the span of the column vectors of $F_{v} G, v \in Q^{<k+1}$. Here we applied Notation 2 to $F_{q}, q \in Q$ to obtain the matrices $F_{v}, v \in Q^{*}$. Define the subspace $\mathscr{I}$ as the space spanned by the column vectors of the matrices $F_{v} G, v \in Q^{*}$. If we can show that $\operatorname{Im} \mathscr{R}_{n-1}=\mathscr{I}$, then the statement of the theorem follows easily.

Indeed, it is easy to see that the linear span of all reachable states of $\Sigma$ equals $\mathscr{I}$, if we set $F_{q}=A_{q}, q \in Q$ and $G=\widetilde{B}$. Moreover, in this case $\mathscr{R}_{n-1}=\mathscr{R}(\Sigma)$. Hence, rank $\mathscr{R}(\Sigma)=n$ is equivalent to $\mathscr{I}=\mathbb{R}^{n}$, which in turn is equivalent to span-reachability of $\Sigma$. Similarly, if we set $F_{q}=A_{q}^{T}$ and $G=\widetilde{C}^{T}$, then $\mathscr{O}(\Sigma)^{T}=\mathscr{R}_{n-1}$ and $\mathscr{I}$ is the orthogonal complement of $\bigcap_{v \in Q^{*}} \operatorname{ker} \widetilde{C} A_{v}$. From [30] it follows that $\Sigma$ is observable if and only if $\bigcap_{v \in Q^{*}} \operatorname{ker} \widetilde{C} A_{v}=\{0\}$, which is equivalent to $\operatorname{Im} \mathscr{R}_{n-1}=\mathscr{I}=\mathbb{R}^{n}$. The latter is equivalent to rank $\mathscr{O}(\Sigma)=n$.

We proceed to show $\mathscr{I}=\operatorname{Im} \mathscr{R}_{n}$. The proof is the same as the one of an analogous statement for rational representations or state-affine systems [18, 29]. We repeat it for the sake of completeness. It is easy to see that $\operatorname{Im} \mathscr{R}_{k} \subseteq \mathscr{I}$ for all $k \in \mathbb{N}$ and $\operatorname{Im} \mathscr{R}_{k} \subseteq$ $\operatorname{Im} \mathscr{R}_{k+1}$. By a dimensionality argument it follows that there exist $0 \leq k_{*} \leq n-1$, such that $\operatorname{Im} \mathscr{R}_{k_{*}}=\operatorname{Im} \mathscr{R}_{k_{*}+1}$. From this, by noticing that $\operatorname{Im} \mathscr{R}_{k+1}=\operatorname{Im} G+\sum_{q \in Q} \operatorname{Im} F_{q} \mathscr{R}_{k}$, it follows that $\mathscr{I}=\operatorname{Im} \mathscr{R}_{k_{*}}$. Since $\operatorname{Im} \mathscr{R}_{k_{*}} \subseteq \operatorname{Im} \mathscr{R}_{n-1}$, we then obtain that $\operatorname{Im} \mathscr{R}_{n-1}=\mathscr{I}$. 